\title{
Long Queue Estimation for Signalized Intersections Using Mobile Data
}

\section{Peng Hao, Ph.D}

Center for Environmental Research \& Technology, University of California, Riverside, 1084 Columbia Avenue, Riverside, CA 92507, USA

Tel: +1 951781 5777, Fax: +1 951781 5790, Email: haop@ cert.ucr.edu

\section{Xuegang (Jeff) Ban, Ph.D*}

Department of Civil and Environmental Engineering, Rensselaer Polytechnic Institute, 110 Eighth Street, 4034 Jonsson Engineering Center, Troy, NY 12180-3590, USA Tel: +1 518276 8043, Fax: +1 518276 4833, Email: banx@rpi.edu

*: Corresponding Author

Resubmittal to Transportation Research Part B September, 2015 


\begin{abstract}
Queue length is one of the key measures in assessing arterial performances. Under heavy congestion, queues are difficult to estimate from either fixed-location sensors (such as loop detectors) or mobile sensors since they may exceed the region of detection, which is defined as long queue in the literature. While the long queue problem has been successfully addressed in the past using fixed-location sensors, whether this can be done using mobile traffic sensors remains unclear. In this paper, a queue length estimation method is proposed to solve this long queue problem using short vehicle trajectories obtained from mobile sensors. The method contains vehicle trajectory reconstruction models to estimate the missing deceleration or acceleration process of a vehicle. Long queue estimation models are then developed using the reconstructed vehicle trajectories. The proposed method can provide estimates of the queue profile and the maximum queue length of a cycle. The method is tested in a field experiment with reasonable results.
\end{abstract}

Key Words: Signalized Intersections; Queue Length Estimation; Queue Profile Estimation; Mobile Sensors; Vehicle Trajectory; Vehicle Acceleration/Deceleration Reconstruction 


\section{Introduction and Motivation}

Arterial performance measures such as queue lengths at signalized intersections and route travel times are crucial means to quantify the performance of arterial systems, and thus have attracted much attention in the literature. For example, there are many applications that would benefit from detailed travel time information through an urban traffic network, such as traffic navigation and fleet management. Arterial traffic flow however is challenging to model or measure. One of the reasons is that intersections bring disruptions to arterial traffic flow, which results in complex traffic evolution over time and space in queues close to intersections and due to traffic signals. Meanwhile, arterial traffic data are increasingly available today. Third party traffic data collectors already started to gather traffic information in real time (Brindle, 2014), e.g., vehicle locations and speeds updated in seconds or a minute, or vehicle travel times for specific routes. This research seeks to gain a deeper understanding using such data to estimate signalized intersection queuing and thus, which can lead to better estimation of arterial performances in real time (and potentially for very large areas).

In the past, queue length estimation for signalized intersections mostly concentrated on the static or average queue lengths using statistical methods (Webster, 1958; Newell, 1965) or shockwavebased methods (Lighthill and Whitham, 1955; Richards, 1956; Stephanopoulos and Michalopoulos, 1979). With the growing demand of live traffic information and the development of new sensing technologies, real time arterial performance, such as the cycle-by-cycle (or real time) queue length, has gained much attention. Such real time traffic studies have been conducted using (i) 30-second loop detector data with fine-grained signal timing (Skabardonis and Geroliminis, 2008); (ii) event-based signal and loop detector data (Balke et al., 2005; Smaglik et al., 2007; Liu and Ma, 2008; Liu et al., 2009); and (iii) other techniques (such as video-based) that have not been seen as widespread use. Mobile traffic sensors, such as Global Position System (GPS), cellular phones, Connected Vehicles, and other tracking devices, provide a supplement or alternative to fixed-location sensors in arterial performance measurement. Mobile data have shown great potential for real time queue length estimation (Ban et al., 2011; Hao et al., 2014a, 2014b; Cheng et al., 2012; Comert and Cetin, 2009; Comert, 2013), along with new challenges (e.g., privacy protection; see Sun et al., 2013).

We note here that fixed-location sensor data and mobile data are complementary to each other. They both have their own advantages and disadvantages. In some areas, there may be only fixedlocation sensors so that previous methods based on fixed-location data (Skabardonis and Geroliminis, 2008; Liu et al., 2009) can be used. In some other areas, there may be only mobile sensing data (e.g., from smart phones or probe/fleet vehicles), for which the method proposed here may be applied. In other areas, both data elements may exist, for which one can fuse them to extract more useful information (e.g., see Sun et al., (2015)). Even when new technologies 
such as Connected Vehicles are widely deployed, fixed-location sensors may still be needed for security or reliable monitoring purposes. In this case, the fixed-location data, especially the event-based data will also be useful sources for measuring the performances of traffic systems such as queue lengths of signalized intersections. In addition, fixed-location sensors have inherent advantages in real time traffic control as they are able to acquire the lane-by-lane incoming flow efficiently. The proposed methods here do not imply that that mobile sensors are superior and will replace fixed-location sensors (such as loops) in the future; rather they provide alternative ways to assess arterial performance measures using mobile data.

In this paper, we focus on mobile sensing data only, and aim to answer the question of what one can do to estimate signalized intersection queue length using mobile data especially when the queue is long. Here we are concerned with queue length estimation, not prediction. Estimation means one infers something (e.g., queue lengths) after the fact, e.g., to infer queue information of a cycle when a vehicle in the cycle passes the downstream location (VTL2; see below) or more practically, to estimate the queue length profile of a cycle when the cycle finishes. One may possibly build prediction models using the estimated results, by modeling how the queues likely change over time. This however is not a trivial task, and not the focus of the paper. Also "queue length" here is defined as the number of vehicles that have been queued in a cycle, which describes how far upstream the queue reaches. This definition was adopted from previous studies (Liu et al., 2009, Ban et al., 2011, Hao et al., 2014b), which was shown to better represent the impact of the queue to the upcoming vehicles (even during the green time period).

For queue length estimation (using either fixed-location sensors or mobile sensors), there is the so-called "long queue" problem (Liu et al., 2009) when the queue exceeds the area of detection (the advanced detector if fixed-location sensors are concerned or the upstream virtual detector, i.e., VTL1 as will be shown later in this paper, if mobile sensors are concerned). Recently such problem was addressed successfully using fixed-location sensors. Skabardonis and Geroliminis (2008) used flow and occupancy as the key measures to estimate the time when long queue or spillover happens. Some adjustments were then applied to estimate the queue length. Liu et al. (2009) identified a long queue by searching the "break points" from the detector occupancies and vehicle gaps. A shockwave-based model was then developed to reconstruct the shockwave propagation beyond the advance detector to recover the length of a possible long queue.

Mobile sensors can collect sample trajectories from vehicles equipped with such sensors. The long queue problem may not be that severe for mobile-data-based models: the queue far from the intersection could still be detected by mobile sensors if one assumes the entire trajectories in the upstream link (defined as long trajectories in this paper) of sample vehicles are available for traffic analysis. Some intersection models were built upon those long trajectory data. Izadpanah et al. (2009) proposed a trajectory based shockwave detection model, and tested their model in simulation using long trajectories of all passing vehicles. Cheng et al (2012) developed a cycleby-cycle queue length estimation method using long vehicle trajectories. Critical points were 
extracted from the trajectories which indicate when vehicles change their dynamics (such as slow down, speed up, or stop). A shockwave-based model was then proposed to estimate the real time queue length and signal timing using measures at the critical points. Collecting long trajectory data however may violate privacy because the continuous trace of a vehicle may indicate the home and work place of the driver. This may be true even if the entire trajectory is divided into multiple pieces (e.g., one for each of the links between two intersections) since there are methods that can effectively connect those partial, short traces even when they are anonymously collected (Sun et al., 2013).

To protect privacy while still preserving the needed data for traffic modeling applications, Hoh et al. (2008) introduced the concept of Virtual Trip Lines (VTLs). VTLs are "virtual loop detectors" that receive the locations, times, and speeds of passing vehicles. For arterial performance measurement, a pair of VTLs is installed at the upstream and downstream of an intersection (called VTL1 and VTL2 respectively) so that only sample travel times and/or short trajectories within the VTL zone (the region between the pair of VTLs) can be collected. It was shown that those short trajectories can still protect privacy to a reasonable level if VTLs are appropriately deployed and the collected traces are filtered using proper filtering algorithms (Zan et al., 2011; Sun et al., 2013). Ban et al. (2011) showed that travel times collected via the arterial VTL system can be used to estimate real time queue lengths. Hao et al. (2014a) developed a kinematic equation based method to investigate the queue location of each sample vehicle, which also provides a lower bound to the queue length in a cycle. Hao et al. (2014b) also proposed a Bayesian Network based algorithm to evaluate the cycle-by-cycle queue length distribution using sample travel times based on the vehicle index estimation model in Hao et al. (2013). Those methods are all developed upon the assumption that queues never spill back to VTL1. If queue spillback does happen (i.e., the long queue problem), however, such methods cannot be applied directly to estimate long queues, for two reasons.

First, mobile sensors only collect traces of sample vehicles. Thus most previous studies revealed the hidden information of undetected vehicles by applying certain arrival assumptions, e.g. uniform arrival assumption in Ban et al. (2009, 2011) and Cheng et al. (2012), non-homogeneous Poisson arrival assumption in Hao et al. (2014b). All these assumptions consider the arrival traffic as free flow which is not disturbed by the signals or queues. In the long queue case, the free flow arrival assumption will not hold if the arrival flow at VTL1 is impacted by the queue. If vehicles are queued before entering the VTL zone, they may pass VTL1 with the saturation flow rate, instead of the actual arrival flow rate. Second, for the queue estimation method in Ban et al. (2011) and Hao et al. (2014a, 2014b), sample travel times are directly obtained and used. The measured intersection delay is the key measure to assess whether a vehicle is queued or not. The short queue assumption is introduced to ensure that the delay experienced during the deceleration, queuing, and acceleration process is fully captured by the two VTLs. If vehicles are queued before entering the VTL zone, the measured delays do not agree with the exact delays 
experienced by the vehicles. Errors may thus occur if such measured delays are directly used to identify whether vehicles are queued or not.

Since VTLs are virtual detectors, one could detect when long queue happens and then dynamically move VTL1 upstream beyond the end of the queue. This way the long-queue problem will become a short-queue problem. Although this sounds reasonable in theory, for practical reasons, once a VTL is deployed, one would not expect to change it very frequently. Doing so will require the server and clients update the new VTL location frequently which may impose extra computational burden to the system. This could also bring inconsistencies for certain applications that rely on specific VTL locations before and after the VTL is updated. Even one could move a VTL farther upstream once the system detects that the queue exceeds VTL1, from the time this is detected to when the VTL can be replaced and updated, there might be some delays (say a few minutes or more). During this period, this long queue problem still exists. In this case, a method that can directly estimate the length of a long queue using mobile data is still preferable. The long queue methods previously developed using fixed-location data (Liu et al., 2009; Skabardonis and Geroliminis, 2008) may not be applied here since they require vehicle occupancies or gaps as the input, which cannot be measured directly by mobile sensors.

In this paper, we aim to solve the long queue problem using short vehicle trajectories collected in a VTL zone from mobile sensors. A vehicle trajectory reconstruction method is first developed to recover the free flow arrival traffic state from short trajectories when long queue happens, based on which to estimate the total intersection delay of a sample vehicle. Separate models are built upon different cases when a vehicle passes VTL1 (i.e., the upstream VTL). If the vehicle passes VTL1 in the deceleration process, the simplified car following model in Newell (2002) will be applied. The vehicle is assumed to follow the trajectory of the leading vehicle, with some shifts in time and space. Thus the missing part in the deceleration process can be trained/estimated by the sample queued vehicles in the dataset. For a vehicle that passes VTL1 during acceleration, we develop a queue dissipation model based on the LWR theory (Lighthill and Whitham, 1955; Richards, 1956) to reconstruct its trajectory up to the point when the vehicle stops in the queue. After the missing vehicle trajectory is reconstructed, the long queue problem will be reduced to a short queue estimation problem that can be solved using existing methods, e.g, the one in Ban et al. (2011). Here we develop a delay-based method for the short queue problem to estimate the cycle-by-cycle queue length profile and the maximum queue length. Both the trajectory reconstruction and queue length estimation methods are tested and validated using field data, with reasonable results. Compared with previous studies conducted by the authors (Ban et al. 2011, Hao et al. 2014a, 2014b), this paper introduces the short vehicle trajectories as the major input instead of travel times to tackle the long queue problem. The major contributions of the paper are summarized as follows: 
(1) The missing (i.e., undetected) deceleration process of a sample vehicle is reconstructed by applying the simplified car following model proposed previously in Newell (2002). This enables us to estimate the queue profile and the maximum queue length of the cycle.

(2) The missing acceleration process of a sample vehicle is reconstructed by applying the firstorder traffic flow theory (Newell, 1993). This enables us to estimate the queue location and position of the sample vehicle, which contributes to the queue profile and also provides a lower bound of the maximum queue length of the cycle.

(3) Models are trained (e.g., the characteristic lines of traffic flow) using sample short vehicle trajectories from multiple cycles, rather than from a single cycle. This can help resolve the issue of small sample size if data from only one cycle are used, which also provides more robust results.

\section{Preliminaries}

We first provide some definitions that will be used throughout this paper, together with some key assumptions that will be applied in developing the trajectory reconstruction and queue length estimation models.

\subsection{Definitions}

- Minimum traverse time (MTT): the shortest time that a (free flow) vehicle passes the VTL zone in the dataset. MTT can be estimated/calibrated using measured data (Ban et al., 2011). MTT is denoted as $\tau_{f}$ in this paper.

- Measured VTL delay: the time difference between the exact travel time and the minimum traverse time within the VTL zone when a vehicle passes the intersection.

- Intersection delay: the total delay a vehicle experiences when it passes the intersection, including the measured VTL delay and the possible delay before VTL1 (for the long queue case).

- No-delay Arrival Time (NAT): the estimated time that a vehicle would have arrived at the stop line if no intersection delay had been experienced (Ban et al., 2011).

- Queued vehicle: a vehicle whose experienced intersection delay exceeds a pre-defined threshold; see Ban et al. (2011).

- Queue length: The number of queued vehicles in a cycle, which indicates how far the queue reaches to the upstream. See Hao et al. (2014b).

- Queue position of a vehicle: the position of the vehicle in the queue. The first vehicle in the queue has the position 1, the second has the position 2, and so on.

- Queue location of a vehicle: the location of the vehicle in the queue. It is measured as the distance of the vehicle in the queue to the stop line. The location of the first queued vehicle is zero.

- Queue length profile of a cycle: the positions (or locations) of all vehicles in the cycle, plotted with respect to the times that they join the queue. 


\subsection{Assumptions}

(1) All vehicles travel at free flow state before they slow down by the queue at the intersection.

(2) Same deceleration behavior of the first vehicle in the queue in response to the red light. This is true regardless of the specific cycle the vehicle is in. The trajectories of the other queued vehicles in the same cycle will be the same as the first queued vehicle with appropriate displacement in time and space; see Newell (2002).

(3) The signal timing information may not be known.

(4) Queue from the downstream intersection does not reach to VTL2.

\subsection{Overview of Long Queue Estimation}

Figure 1 depicts the vehicle trajectories in the field test conducted in Albany, NY for about 3 minutes (Ban et al., 2011). The $y$-axis represents the distance to the stop line (positive values indicate the upstream locations of the stop line). As about $30 \%$ vehicles were equipped with mobile sensors, we show the entire trajectories of those sample vehicles in the figure. If VTL1 is far enough, e.g. $350 \mathrm{ft}$ from the stop line as indicated by the horizontal bold solid line, the arrival flow can be considered as free flow and the VTL zone captures the complete delay process of queued vehicles. If the upstream VTL is close to the stop line, say $150 \mathrm{ft}$ as shown by the horizontal bold dashed line, the free flow arrival assumptions cannot be applied directly because the incoming flow at VTL1 is disturbed by the undetected queue (circled in the figure). Moreover, for the long queue case, the measured delay may not indicate the exact queuing state of the vehicle. For example, vehicle $e$ is apparently a queued vehicle, but will be mistakenly identified as a free flow vehicle based on its measured VTL delay since the vehicle is queued before entering VTL1. Therefore, it is a critical task in the long queue model to reconstruct the missing trajectory of a queued vehicle and estimate its missing delay that cannot be fully 


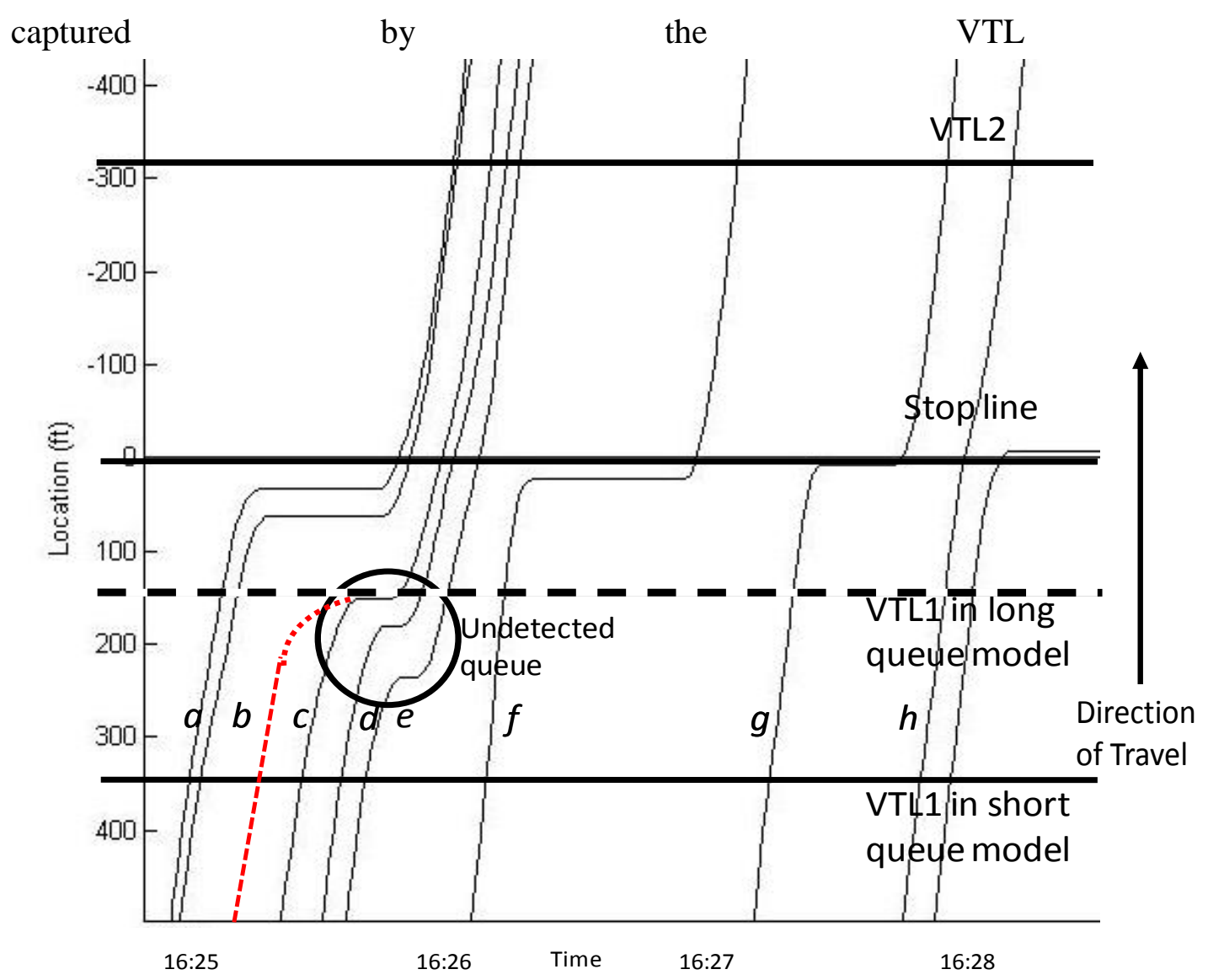

Figure 1 Long queue in the field test data

As shown in Figure 1, the intersection delay of each queued vehicle (i.e. vehicle $a-g$ ) consists of three parts: the deceleration delay, queuing delay (defined as the waiting time when the vehicle stops) and the acceleration delay. The free flow vehicles (i.e. vehicle $h$ ) do not have any delays. For each vehicle, we first figure out whether the vehicle is queued and which part of delay is missing (if queued) based the short trajectory captured by the VTL zones. There are four possible types of vehicles in the long-queue case:

Type I vehicle (vehicle $f$ and $g$ ): a vehicle that passes VTL1 with free flow speed, and then slows down, stops and accelerates to free flow speed within the VTL zone. For this type of vehicle the measured VTL delay is exactly the same as the intersection delay.

Type II vehicle (vehicle $a$ and $b$ ): a vehicle that enters the VTL zone when decelerating, and then stops in the queue and passes the intersection. In this case, the deceleration process before VTL1 is not available directly, but can be reconstructed based on the simplified car-following model in Newell (2002).

Type III vehicle (vehicle $c$ and $d$ ): a vehicle that enters the VTL zone when accelerating, and leaves the intersection without stop. As the deceleration and queuing process beyond the VTL 
zone is not observed, the short trajectory in the VTL zone is not sufficient to determine the waiting time in the queue. For example, if vehicle $c$ arrives 10 seconds earlier, it might still stop at the same location and then follow the same acceleration trajectory. As a result, if only the acceleration process is observed, the hidden trajectory before VTL1 can be either the ground truth (solid line) or the dashed curve which arrives 10 seconds earlier.

Type IV vehicle (vehicle $e$ and $h$ ): a vehicle that passes the VTL zone with free flow speed. It can be either a true free flow vehicle (i.e., $h$ ), or a queued vehicle which stops and accelerates to free flow speed before VTL1 (i.e., e). We cannot acquire any queue information for the type IV vehicle due to the ambiguity in identifying the vehicle queuing status. The methods presented here therefore cannot correctly identify Type IV vehicles; they are simply considered as free flow vehicles and will not be used for queue length estimation in this paper.

Type I vehicles are actually captured by the short queue case; see e.g., Ban et al. (2011). For a Type I vehicle, its queue position and queue location can be estimated directly; see Section 3 below. For a Type II or III vehicle, the queuing information is not completely revealed due to the short distance between VTL1 and the stop line. However, we are able to reconstruct the missing trajectories based on the observed mobile data and transportation knowledge. Therefore, the long-queue estimation methods for Type II and Type III vehicles consist of two major steps: the missing deceleration (for Type II vehicles) or acceleration (for Type III vehicles) process reconstruction and long-queue estimation. We call the first step the trajectory reconstruction. It turns out that after trajectory reconstruction, the long queue problem can be reduced to a short queue problem for both Type II and Type III vehicles. As a result, the queue position and queue location of a type II or Type III vehicle can also be estimated after the trajectory reconstruction step; see Section 4 and Section 5 respectively. The estimated queue positions (or locations) of all sample queued vehicles in a cycle essentially provide the profile of the queue in the cycle. In addition, the maximum queue length of a cycle can be inferred from the last sample queued Type I or Type II vehicle. This cannot be done for the last queued Type III vehicle, whose queue position however does provide a lower bound of the maximum queue length of the cycle. Therefore, if a cycle contains multiple types of vehicles, the maximum queue length of the cycle can be obtained as the largest of the maximum queue lengths estimated from the last sample queued Type I and Type II vehicles and the queue position of the last sample queued Type III vehicle. As aforementioned, a Type IV vehicle will be considered as a free flow vehicle in this paper which does not directly contain information about queues.

The four vehicle types we presented above only cover normal cases, i.e., the queued vehicles are fully stopped (a standing queue) which can be cleared in one cycle. In real world situations, a queued vehicle may never fully stop - the so-called moving queue. It turns out that the longqueue estimation methods for moving queue are quite similar to the methods discussed in Sections 3 - 5 below. In addition, over-saturation may happen under heavy congestion when a queue cannot be fully discharged in one cycle and has to wait for extra red times. Details for long 
queue estimation for moving-queue and over-saturated vehicles are provided in the appendix of this paper.

\section{Delay-based Short Queue Length Estimation for Type I Vehicles}

In this section, we focus on Type I vehicles, such as vehicle $f$ or $g$ in Figure 1. According to the delay pattern model in Ban et al. (2009), the intersection delay drops approximately linearly in a cycle. The time that the delay reduces to zero helps to estimate the maximum queue length of the cycle, assuming the discharge shockwave speed and departure shock speed are known (Ban et al., 2011). As those delay-based models use sample travel times as the input, and ignore the delays in acceleration and deceleration, we cannot directly apply them to estimate the queue length from short trajectories. However, the idea of the delay pattern based models is adopted in this paper, such as the delay-based definition of queues and the uniform arrival assumption as mentioned in Section 2.1 and 2.2. The proposed algorithm requires the trajectory of the Type I vehicle as the input, along with some pre-defined (or pre-learned) parameters, such as average jam density, arrival headway and saturation headway.

First, the queue location of the $i$-th sample queued Type I vehicle (denoted as $L_{i}$ ) can be directly measured from the sample trajectory. If we assume that queued vehicles are queued with intersection-specific jam density $k_{j}$, the queue position of the vehicle expressed in number of vehicles is

$$
Q_{i}=k_{j} L_{i}+1
$$

This is because the queue location of the $1^{\text {st }}$ vehicle in a queue is assumed to be 0 . As aforementioned in Section 2.3, the locations and positions of all sample queued Type I vehicles in a cycle can contribute to the profile of the queue in the cycle. Notice that if there is no sample queued Type I vehicle, we set $Q_{i}=0$ directly.

To determine the maximum queue length of a cycle with sample queued Type I vehicles, we focus on the last sample queued Type I vehicle. The NAT of this vehicle (denoted as $t_{N A T}^{l}$ ) can be derived from its arrival time at VTL1 (denoted as $t_{1}^{l}$, which can be directly obtained from its trajectory) by adding the minimum traverse time from VTL1 to the stop line (denoted as $\tau_{f}$ ), i.e., $t_{N A T}^{l}=t_{1}^{l}+\tau_{f}$. Assume that vehicles arrive with the average headway $h_{a}$ and discharge with the average saturation headway $h_{s}$, the intersection delay will reduce at a constant reduction rate $h_{a}-h_{s}$ for two consecutively queued vehicles. Thus, the vehicle queue position difference between the last sample queued vehicle and the exact queue rear is estimated by $\frac{t_{S L}^{l}-t_{N A T}^{l}}{h_{a}-h_{S}}$, where $t_{S L}^{l}$ is the departure time of the last sample queued Type I vehicle at the stop line. The maximum queue length of the cycle (denoted as $Q_{m}$ ) is obtained by adding this increment to the queue position of the last sample queued Type I vehicle (denoted as $Q_{l}$ which can be calculated using equation (1)): 


$$
Q_{m}=Q_{l}+\frac{t_{S L}^{l}-t_{N A T}^{l}}{h_{a}-h_{s}}=k_{j} L_{l}+\frac{t_{S L}^{l}-t_{N A T}^{l}}{h_{a}-h_{s}}+1
$$

As shown in equation (2), the NAT $t_{N A T}^{l}$, departure time at the stop line $t_{S L}^{l}$, and queue location $L_{l}$ of the last sample queued Type I vehicle are three key variables for the maximum queue length estimation. For Type I vehicles, they can be directly measured from the vehicle trajectory. For a Type II vehicle, as the deceleration process starts before VTL1, we have to estimate the NAT based on the reconstructed trajectory. After the NAT of the last sample queued Type II vehicle is estimated, we are able to apply the same method in (2) to estimate the maximum queue length, in addition to the positions and locations of all Type II vehicles in the queue. The details will be provided in Section 4. For a Type III vehicle, as discussed in the previous section, it is impossible to determine the no-delay arrival time or exact intersection delay. Therefore, (2) cannot be applied to Type III vehicles. However, we can use the observed incomplete acceleration process to reconstruct the entire acceleration process to estimate the queue location and position of a Type III vehicle. The queue position of the last sample queued Type III vehicle in a cycle can provide a lower bound of the maximum queue length of the cycle. This is presented in Section 5.

In equation (2), the average arrival headway $h_{a}$ which indicates the traffic condition is roughly estimated using the sample data and penetration rate. For a certain time period, say $T$ seconds, assume the sample size is $N$ and the penetration rate is about $p$, we can estimate the average arrival headway using the following equation $h_{a}=\frac{p T}{N}$. Note that the exact penetration rate is not available for many cases. If the penetration rate is unknown, we can estimate it by computing the percentage of the sample queued vehicles in the total queued vehicles. The penetration rate estimation algorithm is provided in the appendix of Hao (2013). The saturation headway $h_{s}$ is more stable and independent of time. If there is some historical data at the same intersection, we can use them to estimate the average saturation headway when vehicles are discharged from the queue. If the historical data is not available, we take a predefined value, say 2 seconds, as the average saturation headway.

\section{Queue Length Estimation for Type II Vehicles}

Similar to a Type I vehicle, the queue location of a Type II vehicle is revealed directly in its short trajectory in the VTL zone. The queue position of a Type II vehicle can also be estimated using equation (1). In this section, we focus on the maximum queue length estimation of Type II vehicles. First we reconstruct the full deceleration process of a Type II vehicle using the simplified car following model in Newell (2002). The detected deceleration trajectories of all vehicles are archived for training. This will provide a way to estimate the NAT of the last sample queued vehicle. The maximum queue length can then be estimated based on this estimated NAT using equation (2). 


\subsection{Deceleration Process Reconstruction}

We aim to reconstruct the hidden deceleration process of Type II vehicles. Here we assume there is interaction among Type II vehicles since they all approach the intersection and are queued. We apply in this paper, the simplified car following model proposed in Newell (2002). According to the model, the following vehicle has essentially the same time-space trajectory as the leading vehicle except for shifts in time and space on a homogeneous highway. Newell also mentioned the application of the simplified model to signalized intersections. When the signal turns red, the first vehicle decelerates and stops at the stop line. Then all queued vehicles in this cycle will approximately follow the same trajectory as the first one, with lag in time and displacement in space. We further assume the same deceleration behavior of the first vehicle in any cycle approaching the intersection. Thus, all queued vehicles arrived at a specific intersection will follow the same trajectory with time and space shifts.

Let $x=f(t)$ be the time-space trajectory function of some given queued vehicle (say the first queued vehicle in the data set), where $x$ is the distance from the vehicle to the stop line, and $t$ is the exact time. The trajectory of any other queued vehicle would be a transition of the function with appropriate constants that represent the space and time translates.

$$
x+x^{*}(A)=f\left(t+t^{*}(A)\right)
$$

where $A$ is the cumulative flow (or vehicle number) of the subject vehicle. $x^{*}(A)$ and $t^{*}(A)$ represent the shift of time and space of the vehicle respectively. Notice that the cumulative flow (Newell, 1993) is defined as "the cumulative number of vehicles to pass location $x$ by time $t$ starting from the passage of some reference vehicle." If the first-in-first-out (FIFO) rule is satisfied, the A-curve of an integer cumulative flow (say $N$ ) corresponds to the trajectory of the $N^{\text {th }}$ vehicle after the reference vehicle. The concept of cumulative flow was widely applied in signalized intersection traffic research (Liu et al, 2008).

The speed of the vehicle at $(t, x)$ is then

$$
u=-\frac{\partial x}{\partial t}=-f^{\prime}\left(t+t^{*}(A)\right)(4)
$$

Here $f^{\prime}(t)$ denotes the full derivative of the function $f$ over its defining variable $t$, i.e., $\frac{d f}{d t}$. Note that we use minus sign in the expression, as the vehicle is traveling in the opposite direction to the direction of the $\mathrm{x}$-axis; see Figure 1. As the speed is a strictly monotonic decreasing function of time in deceleration, we can always find a function $g(*)$ that is the inverse function of $-f^{\prime}(*)$, and rewrite function (4) as follows

$$
t+t^{*}(A)=g(u)
$$


Thus the time and space coordinate of any trajectory point in deceleration can be expressed as functions of cumulative flow $A$ and speed $u$

$$
\left\{\begin{array}{l}
t(u, A)=g(u)-t^{*}(A) \\
x(u, A)=f(g(u))-x^{*}(A)
\end{array}\right.
$$

Equation (6) shows that the trajectory point of a vehicle $(t, x)$ can be determined by the cumulative flow and the vehicle speed, during the vehicle deceleration process. In other words, given the vehicle (e.g. the $A$ number is known), the trajectory of the vehicle can be solely determined by the speed of the vehicle.

Now we focus on the trajectory of the $A^{* \text { th }}$ single vehicle. Along this trajectory, we have

$$
\left\{\begin{array}{l}
t\left(u, A^{*}\right)=g(u)-t^{*}\left(A^{*}\right) \\
x\left(u, A^{*}\right)=f(g(u))-x^{*}\left(A^{*}\right)
\end{array}\right.
$$

Take the first derivative of equation (7.1), we have

$$
\frac{d t}{d u}=g^{\prime}(u)
$$

Clearly $g^{\prime}(u)$ is the reciprocal of the vehicle's acceleration/deceleration rate based on equation (8). For each vehicle, the value of the function $g^{\prime}(u)$ at any speed $u$ can be derived from the trajectory by measuring the change of time in respect to the unit change of speed. We can compute the average value of $g^{\prime}(u)$, i.e., $\bar{g}^{\prime}(u)$, of all vehicles (over all cycles since we assume same deceleration behavior of all vehicles) that decelerates to speed $u$ within the VTL zone to obtain a robust result.

As the time and speed that the vehicle passes VTL1 is measured, equation (7) also applies to speed $u_{1}$ :

$$
\begin{aligned}
& t\left(u_{1}, A^{*}\right)=t_{1}(9.1) \\
& x\left(u_{1}, A^{*}\right)=x_{1}(9.2)
\end{aligned}
$$

where $u_{1}$ and $t_{1}$ are the speed and time measured at VTL1, and $x_{1}$ is the distance from VTL1 to the stop line. It turns out that (9) can be considered as the initial condition when solving equations (7) and (8) for the vehicle trajectories. The corresponding trajectory point in the deceleration process at any speed $u$ is computed using the calibrated function $\bar{g}^{\prime}(u)$ and the measurements at VTL1, as follows

$$
t\left(u, A^{*}\right)=t_{1}+\int_{u_{1}}^{u} \bar{g}^{\prime}(v) d v(10.1)
$$




$$
x\left(u, A^{*}\right)=x_{1}-\int_{u_{1}}^{u} v \bar{g}^{\prime}(v) d v(10.2)
$$

This equation provides a feasible approach to reconstruct the missing deceleration process of a queued vehicle based on the short sample trajectories within the VTL zone.

\subsection{Maximum Queue Length for Type II Vehicles}

As stated in equation (2), the delay-based maximum queue length estimation requires the NAT, departure time and queue location of the last sample queued vehicle. If the last sample queued vehicle is a Type II vehicle, the departure time and queue location can still be measured directly from its trajectory. The NAT however needs to be estimated. This can be done if we can find a trajectory point with free flow speed in the reconstructed departure process. Therefore, by substituting free flow $u_{f}$ into equation (10), we can trace the vehicle trajectory back to the timespace coordinate $\left(t_{f}, u_{f}\right)$ that the vehicle starts to decelerate from free flow. This way we can estimate the NAT of this Type II vehicle by plotting an imaginary free flow vehicle trajectory that passes $\left(t_{f}, u_{f}\right)$. This is shown in Figure 2.

Figure 2 illustrates the exact trajectory of a Type II vehicle using the solid curve, and the imaginary free flow vehicle trajectory using dash line. The NAT of the vehicle is computed by

adding the free flow travel time $\frac{x_{f}-x_{1}}{u_{f}}$ and the minimum traverse time $\tau_{f}$ to the free flow arrival time $t_{f}$ :

$$
t_{N A T}=t_{f}+\frac{x_{f}-x_{1}}{u_{f}}+\tau_{f}
$$

After the NAT is estimated, the maximum queue length of the cycle can be estimated by substituting the derived NAT $t_{N A T}$, the measured departure time $t_{S L}$, and the queue location $L_{l}$ of the last sample queued Type II vehicle to equation (2). 


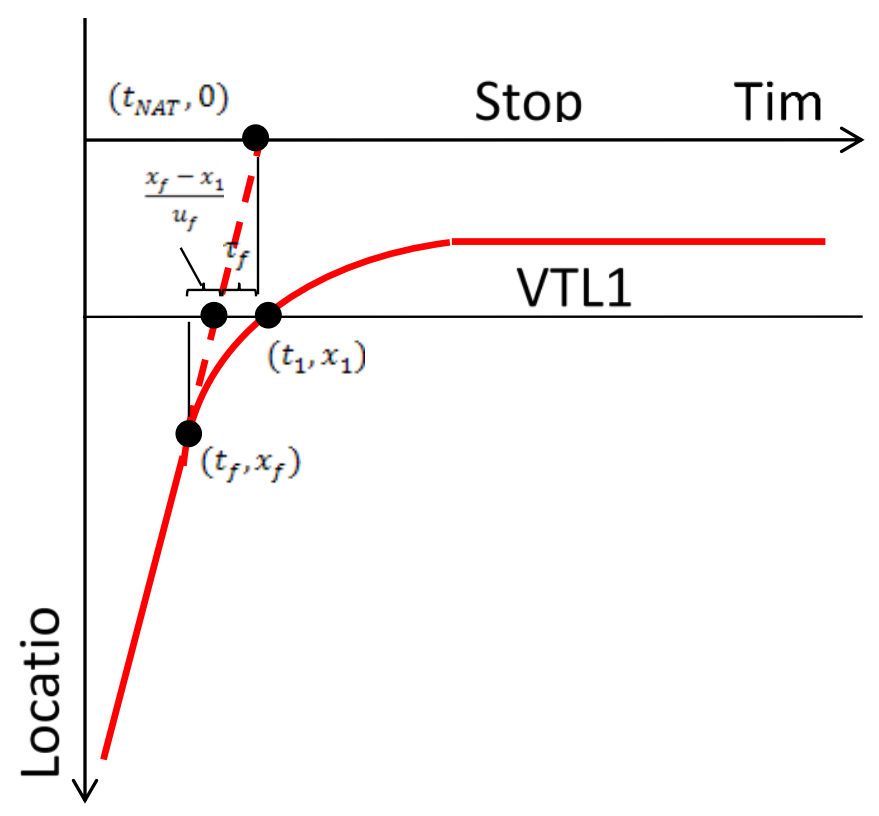

Figure 2 NAT Estimation of Type II Vehicle

\section{Queue Length Estimation for Type III Vehicles}

In this section, we develop a traffic flow theory based estimation algorithm to reconstruct the vehicle acceleration process, based on which to estimate the queue location of a Type III vehicle. This algorithm uses the acceleration trajectories of all Type I and II sample vehicles for training, and works with or without signal timing information. Using the estimated queue location information, equation (1) can be used to estimate the queue position of the Type III vehicle, which can be used to estimate the queue profile of the cycle. The queue position of the last sample queued Type III vehicle in a cycle then provides a lower bound of the maximum queue length of the cycle.

\subsection{Characteristic Curve and Cumulative Flow}

Based on the LWR theory (Lighthill and Whitham, 1955; Richards, 1956), the density $k$ and the flow rate $q$ are associated by the conservation equation (i.e., the continuity equation)

$$
\frac{\partial q(t, x)}{\partial x}+\frac{\partial k(t, x)}{\partial t}=0
$$

For a homogeneous road section, the flow rate $q$ is a function of density $k$ for some given function $f_{q}$

$$
q(t, x)=f_{q}(k(t, x))
$$

As $f_{q}(k, t, x)=f_{q}(k)$, this function is independent of $x$ and $t$. 
Substitute equation (13) into equation (12), we derive a partial differential equation of $q(x, t)$

$$
\frac{d f_{q}}{d k} \cdot \frac{\partial k(t, x)}{\partial x}+\frac{\partial k(t, x)}{\partial t}=0
$$

Hence $q(t, x)$ remains constant along a straight characteristic curve defined by the following differential equation (Newell, 1993)

$$
\frac{d x}{d t}=\frac{d f_{q}}{d k} \equiv w(15)
$$

where $w$ is the wave speed at flow rate $q$ and density $k$, which can be also considered as the slope of the tangent at point $(k, q)$ in the flow-density fundamental diagram.

Here we focus on the departure process of a Type III vehicle, which corresponds to the right branch of the flow-density fundamental diagram (the segment from the jam state to the saturation state). To ensure all traffic state measures (e.g., $q, k, u, w$ ) have one-to-one correspondence with each another, we assume that the right branch of the fundamental diagram is concave, although the exact form of $f(k)$ is not specified. Furthermore, we use speed $u$ to represent the state as it is easy to obtain from mobile data. Then the flow rate $q$, density $k$ and wave speed $w$ can all be expressed as functions of the speed $u$ independent of $x$ and $t$.

\subsection{Queue Dissipation Model}

Based on the definitions and assumptions in the last subsection, we present the queue dissipation model at a signalized intersection. Figure 3(a) illustrates the characteristic lines and cumulative flow curves (i.e. vehicle trajectories) in the green phase of a cycle. We define $t=0$ as the start of the effective green time, and $x=0$ as the location of the stop line. The first vehicle that passes the stop line at the beginning of the green phase is denoted as the reference vehicle. Then the characteristic lines emanate from the boundaries defined by the curve $A(t, x)=0$, which corresponds to the trace of the first vehicle in the cycle. Each characteristic line is for a specific speed of the traffic flow. The relationship between vehicle trajectory and the continuous kinematic wave stream was well studied using variational formulation models in Daganzo (2005a, 2005b, 2006). In variational formulation models, the kinematic wave problems are converted to a discrete network problems, and the vehicle numbers is solved by shortest path algorithm. The proposed model is also inspired by the queue discharging model in Stephanopoulos and Michalopoulos (1979). However, the boundary condition in this research is different from the earlier model. In Stephanopoulos' model, initial traffic states (i.e., $q$ and $k$ ) are assigned along the stop line. However, such initial states cannot be directly measured using mobile sensors. In our model, we define the boundary as the first vehicle's trajectory because it is more compatible with mobile data, especially if short vehicle trajectories are available between VTL1 and VTL2. 

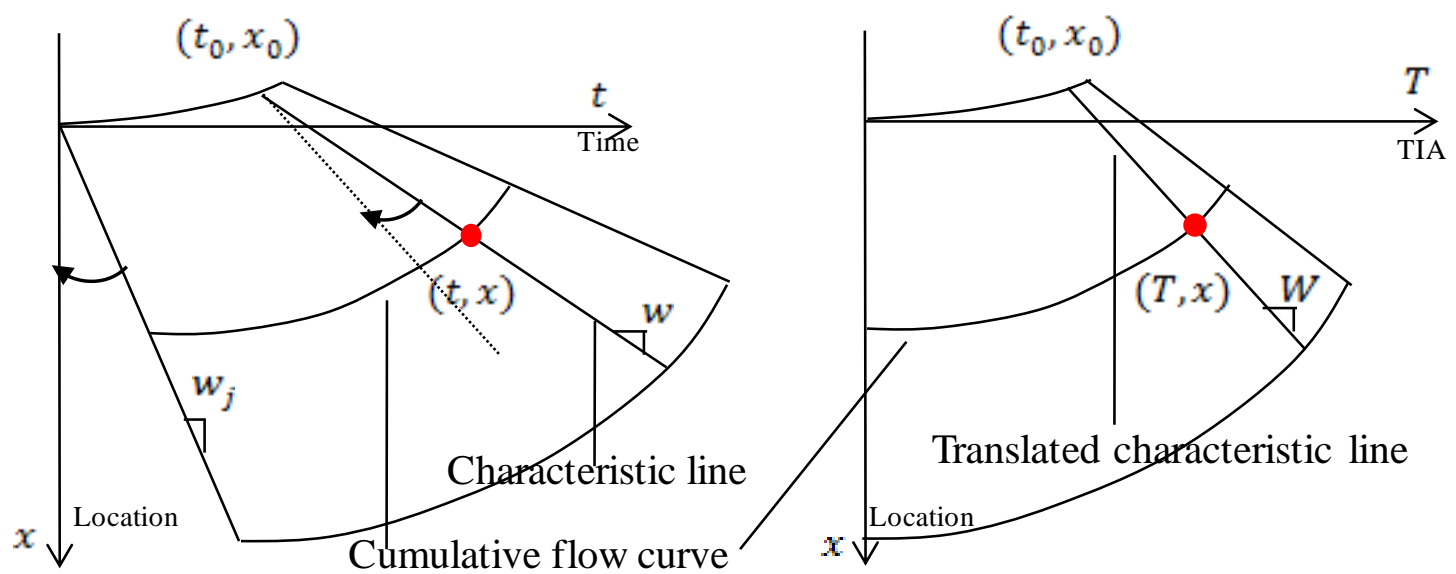

(a) Origin queue dissipation model

(b) The model in translated coordinate system

\section{Figure 3 Queue dissipation model}

Cumulative flow $A$ and speed $u$ provide a new way to interpret the queue dissipation model that is conventionally built on the space-time coordinate system. If $A$ and $u$ are given, one can determine the corresponding $(t, x)$ coordinate because $A$ identifies the vehicle and $u$ specifies the vehicle's time and location in the acceleration process $(u$ monotonically increases in the acceleration process). Next we show this mapping scheme analytically. As stated in Newell (1993), one can compute the change rate of $A$ along the characteristic line of speed $u$ :

$$
\frac{d A}{d t}=\frac{\partial A}{\partial x} \cdot \frac{d x}{d t}+\frac{\partial A}{\partial t}=k w+q(16)
$$

Note that here we use $k, w$ and $q$ for a specific speed $u$, which can be more clearly represented as $k(u), q(u)$ and $w(u)$, which are time independent. Denote $\left(t_{0}, x_{0}\right)$ as the point of given speed on the curve defined by $A(t, x)=0$ (i.e. the trajectory of the reference vehicle). The cumulative flow on the characteristic line of speed $u$ at time $t$ is

$$
A=(k w+q)\left(t-t_{0}\right)(17)
$$

Then the corresponding $(t, x)$ coordinates are specified as follows, calculated along the characteristic line for speed $u$ :

$$
\left\{\begin{array}{c}
t=t_{0}+\frac{A}{k w+q} \\
x=x_{0}+w\left(t-t_{0}\right)=x_{0}+\frac{w A}{k w+q}
\end{array}\right.
$$

Note here that $k, w, q$ are determined by $u$, implying that the trajectory $(t, x)$ can be expressed using $A$ and $u$.

\subsection{Acceleration Process Reconstruction}


We propose a two-step approach to determine where a vehicle stops (note that we cannot determine when it stops as shown in Figure 1), and how it accelerates before entering the VTL zone. The first step is the training stage, for which we parameterize the model by evaluating the functions of characteristic curves at different speeds. The training data include trajectories of all Type I and II sample vehicles. For those vehicles, we can directly acquire the location and speed at any time in the acceleration process. In the second step, we apply the trained model to Type III sample vehicles and reconstruct the missing acceleration process before VTL1.

\subsubsection{Training Characteristic Curves}

According to the traffic flow theory and the feature of mobile sensor data, the proposed approach is built upon two major assumptions. First we assume the road section is homogeneous. Consequently, the characteristic curves are all straight lines and the queue dissipation model in Figure 3(a) can be applied. Next, we assume the boundary condition (i.e. trajectory of the first vehicle in the cycle) and the fundamental diagram (flow/density relationship) do not change in different cycles when we set the start of green time as the reference time and the stop line as the reference location. We note that the first assumption has been widely used in the literature when the LWR theory is applied. The second assumption is applied since we concern about the average (or stationary) traffic flow characteristics. Stochasticity of the traffic flow can be introduced to relax the second assumption if needed; for example, see Hao et al. (2013, 2014b) for stochastic modeling of intersection traffic flow.

The second assumption ensures that the characteristic line of a specific speed in different cycles can perfectly overlay each other if we put them into this reference framework. That is, if the $i^{\text {th }}$ Type I/II vehicle accelerates to speed $u$ at (exact) time $t_{i}$ and location $x_{i}$, then

$$
x_{i}=w\left(t_{i}-T_{g}^{i}\right)+b
$$

where $w$ and $b$ are the slope and intercept of the unique characteristic line of speed $u$, and $T_{g}^{i}$ is the green time start of the cycle that the vehicle leaves the intersection.

In real traffic, we cannot guarantee the equation is hold perfectly for all vehicles due to the driving behavior diversity and measuring error. Thus we apply a simple linear fitting scheme to find the appropriate $w$ and $b$ to minimize the error on time, as follows:

$$
\min _{w, b} \sum_{i=1}^{n}\left(t_{i}-T_{g}^{i}-\frac{x_{i}-b}{w}\right)^{2}
$$

Note that we apply the robust linear regression algorithm here in implementation because the robust model assign different weights to the data points to minimize the impact of outliers. Based on the characteristic line estimation results of different speeds, we are able to derive the functions of the estimated slope and intercept in term of the speed (i.e., $\bar{w}(u)$ and $\bar{b}(u)$ ) 
numerically. Their first derivative, $\bar{w}^{\prime}(u)$ and $\bar{b}^{\prime}(u)$, can be further derived. These trained functions will be used later in acceleration process reconstruction.

In the training stage, one may notice that we build a "cross-cycle" queue dissipation model by introducing the second assumption. That is because we usually deal with low penetration rate data in field tests or real problems. The conventional single cycle model fail to work if a cycle only has zero or one sample vehicle, but the proposed model can aggregate data from sparse samples in different cycles to derive robust results.

\subsubsection{Reconstruction of the Acceleration Process}

As stated, the Type III vehicle stops before entering the VTL zone so that the beginning of its acceleration process is not detected. In this step we aim to find the vehicle queue location where the zero speed characteristic line and the vehicle trajectory intersect. Notice that the zero speed here refers to the actual speed of the traffic, not the wave speed.

We start the discussion with the trained characteristic line functions of some speed $u$

$$
x=\bar{w}(u) t+\bar{b}(u)
$$

Here location $x$ is a function of speed $u$ and time $t$ if $w(u)$ and $b(u)$ are both revealed in the training step. Then the total derivative of $x$ is

$$
d x=\frac{\partial x}{\partial t} d t+\frac{\partial x}{\partial u} d u=\bar{w}(u) d t+\left[\bar{w}^{\prime}(u) t+\bar{b}^{\prime}(u)\right] d u
$$

By the definition of the speed, we have $d x=-u d t$ along the trajectory of the vehicle. Substituting $t=\frac{x-\bar{b}(u)}{\bar{w}(u)}$ and $d t=-\frac{1}{u} d x$ into the equation, the differential equation of $x$ and $u$ is presented as follows: $d x=-\frac{\bar{w}(u)}{u} d x+\left[\bar{w}^{\prime}(u)\left(\frac{x-\bar{b}(u)}{\bar{w}(u)}\right)+\bar{b}^{\prime}(u)\right] d u$. Multiplying $\frac{u d u}{u+\bar{w}(u)}$ on both ends leads to:

$$
\frac{d x}{d u}-\frac{u}{u+\bar{w}(u)} \cdot \frac{\bar{w}^{\prime}(u)}{\bar{w}(u)} x+\frac{u}{u+\bar{w}(u)} \cdot \frac{\bar{b}(u) \bar{w}^{\prime}(u)-\bar{b}^{\prime}(u) \bar{w}(u)}{\bar{w}(u)}=0
$$

Let $H(u)=\frac{u}{u+\bar{w}(u)} \cdot \frac{\bar{w}^{\prime}(u)}{\bar{w}(u)}$ and $Z(u)=\frac{u}{u+\bar{w}(u)} \cdot \frac{\bar{b}(u) \bar{w}^{\prime}(u)-\bar{b}^{\prime}(u) \bar{w}(u)}{\bar{w}(u)}$,

$$
\frac{d x}{d u}-H(u) x+Z(u)=0
$$

This differential equation has an analytical solution

$$
x(u)=-e^{-Y(u)}\left(\int e^{Y(u)} Z(u) d u+C\right)
$$


where $Y(u)=\int H(u) d u$ and $C$ is a constant. The differential equation has a known solution $\left(u_{1}, x_{1}\right)$ if we denote $u_{1}$ and $x_{1}$ as the speed and location collected at VTL1, i.e., the starting point of the collected vehicle trajectory. This solution can be used to determine the constant $C$ in equation (25), which in turn guarantees the uniqueness of the speed-space trajectory function.

\subsection{Model Extension in Absence of Signal Timing}

In the proposed acceleration reconstruction method, cycle-by-cycle signal timing information is a necessary input in the training stage when linearly fitting the characteristic lines from data in different cycles. However, it is not an easy work to collect signal timing information directly from signal controllers (Hao et al, 2012), specifically when the signal is actuated. In this section, we discuss the characteristic curve fitting and acceleration reconstruction approach if the signal timing information is not available.

In the reference framework, we define the reference time as the start of the effective green time. Traffic in different cycles can be normalized to the queue dissipation model as shown in Figure 3(a). Here we introduce a signal timing independent variable, named as Time In Acceleration (TIA), when signal timing information is not available.

For a vehicle which passes location $x$ at time $t$, the TIA at $(x, t)$ is defined as the time elapse from the moment when the vehicle starts to accelerate (denoted as $t_{s}$ ) to the current time $t$. As each Type I/II vehicle has complete acceleration trajectory within the VTL zone, $t_{s}$ can be obtained directly. One can then derive TIA at any trajectory point by subtracting the measured starting time (i.e., $t_{s}$ ) from the current time. Signal information is not required when measuring TIA for Type I or Type II vehicles.

In the queue dissipation model, TIA can be formulated as a function of time and cumulative flow. For some trajectory point $(t, x)$ at which the cumulative flow is $A$, the corresponding starting time $t_{s}$ is the intersection of the trajectory that passes $(t, x)$ and the zero speed characteristic line which can be considered as the interface between the jam state and the acceleration state. On the characteristic line of zero speed $(u=0), t_{0}=0$ and $q=0$. Denote $k_{j}$ as the jam density and $w_{j}$ is the wave speed along the zero speed characteristic line, one can compute the starting time $t_{s}$ from equation (18.1)

$$
t_{s}=t_{0}+\frac{A}{k w+q}=\frac{A}{k_{j} w_{j}}(26)
$$

According to the definition, the TIA (denoted as $T$ ) given $t$ and $A$ is

$$
T=t-t_{s}=t-\frac{A}{k_{j} w_{j}}(27)
$$

Substitute equation (18.1) into (27), 


$$
T=t_{0}+\frac{A}{k w+q}-\frac{A}{k_{j} w_{j}}(28)
$$

This leads to $A=\left(T-t_{0}\right) \frac{k_{j} w_{j}(k w+q)}{k_{j} w_{j}-k w-q}$. Substitute this into (18.2),

$$
x=x_{0}+\frac{w A}{k w+q}=W\left(T-t_{0}\right)+x_{0} \equiv W T+B
$$

where $W=\frac{k_{j} w_{j} w}{k_{j} w_{j}-k w-q}, B=x_{0}-\frac{t_{0} k_{j} w_{j} w}{k_{j} w_{0}-k w-q}$.

As $W$ and $B$ are only dependent on the speed, the vehicle's location is a linear function in term of TIA with speed dependent coefficients. This linear function (29) is denoted as the translated characteristic curve. Figure 3(b) illustrates the queue dissipation model in the translated coordinate system. As shown in the figure, the translated characteristic curve is derived from the corresponding characteristic curve after rotating around $\left(t_{0}, x_{0}\right)$. At zero speed, the translated characteristic line is emanated along the location axis, as TIA is always 0 at the start of the acceleration. Accordingly, the cumulative flow curve (i.e. trajectory) of a given vehicle moves horizontally against the direction of time axis.

We can then apply the proposed characteristic curve fitting method to estimate $\bar{W}(u), \bar{W}^{\prime}(u), \bar{B}(u)$ and $\bar{B}^{\prime}(u)$ from the measured TIA and location data in different cycles, if the second assumption in the previous subsection holds.

Next we will discuss the TIA based approach to estimate the queue location of Type III vehicle. Take the derivative of function (27),

$$
\frac{d T}{d t}=\frac{\partial T}{\partial A} \cdot \frac{d A}{d t}+\frac{\partial T}{\partial t}=-\frac{1}{k_{j} w_{j}} \cdot \frac{d A}{d t}+1(30)
$$

Along the vehicle trajectory with constant cumulative flow, we have $\frac{d A}{d t}=0$ and $\frac{d x}{d t}=-u$, thus

$$
d T=d t=-\frac{1}{u} d x
$$

On the other hand, we take the total derivative of $x$ in function (29),

$$
d x=\frac{\partial x}{\partial T} d T+\frac{\partial x}{\partial u} d u=\bar{W}(u) d T+\left[\bar{W}^{\prime}(u) T+\bar{B}(u)\right] d u
$$

Substitute equation (29) and (31) into (32), we formulate the differential equation of $u$ and $x$,

$$
\frac{d x}{d u}-\frac{u}{u+\bar{W}(u)} \cdot \frac{\bar{W}^{\prime}(u)}{\bar{W}(u)} x+\frac{u}{u+\bar{W}(u)} \cdot \frac{\bar{B}(u) \bar{W}^{\prime}(u)-\bar{B}^{\prime}(u) \bar{W}(u)}{\bar{W}(u)}=0
$$


We can solve this equation with the same method as the signal-based system:

$$
x(u)=-e^{-\bar{Y}(u)}\left(\int e^{\bar{Y}(u)} \bar{Z}(u) d u+C\right)
$$

where $\bar{Y}(u)=\int \frac{u}{u+\bar{W}(u)} \cdot \frac{\bar{W}^{\prime}(u)}{\bar{W}(u)} d u$ and $\bar{Z}(u)=\frac{u}{u+\bar{W}(u)} \cdot \frac{\bar{B}(u) \bar{W}^{\prime}(u)-\bar{B}^{\prime}(u) \bar{W}(u)}{\bar{W}(u)}$

It can be shown that (34) has a unique solution if the location $x_{1}$ and speed $u_{1}$ at VTL1 are collected from a Type III vehicle. The proposed method reconstructs the acceleration process by computing the trajectory point up to the location where the vehicle speed is zero, i.e., the queue location of the vehicle. In practice, we solve this problem numerically by discretizing the speed into small intervals.

\subsection{Queue Location and Position Estimation of A Type III Vehicle}

For a Type III vehicle, we can find the queue location from the reconstructed acceleration trajectory by computing function (34) at zero speed, i.e., $x(0)$. Then the estimated queue position of the vehicle can be obtained using equation (1). The estimated locations (or positions) of all Type III vehicles in a cycle contribute to the profile of the queue in the cycle. The maximum queue length of the cycle however cannot be estimated directly using equation (2) based on Type III vehicles since the NAT cannot be properly estimated for a Type III vehicle. However, the queue position of the last sample queued Type III vehicle, denoted as $Q_{l}^{3}$ does provide a lower bound of the maximum queue length of the cycle. $Q_{l}^{3}$ can be determined as follows based on (1):

$$
Q_{l}^{3}=k_{j} L_{l}^{3}+1
$$

Here $L_{l}^{3}$ is the queue location of the last sample queued Type III vehicle as determined by equation (34). Notice that if there is no sample queued Type III vehicle, we set $Q_{l}^{3}=0$ directly.

\section{Numerical Results}

We summarize the long queue estimation method here. First, the queue location (or position) of each sample queued vehicle (regardless of its type) in a cycle will be estimated to construct the profile of the queue in the cycle. Second, if a cycle does have a mixture of different types of vehicles, the maximum queue length of the cycle will be the maximum value of (i) the estimated queue lengths by equation (2) for Type I and Type II vehicles; (ii) the queue position of the last sample queued Type III vehicle calculated using equation (35); and (iii) the maximum queue length or queue position of moving-queue / over-saturated vehicles estimated based on the discussion in the appendix.

We note there that the proposed method is lane-specific, which however could also solve the queue length problem for multiple lanes if lanes can be properly identified. This does not require the mobile data are accurate enough to distinguish lanes directly. For example, on facilities with 
separate queues such as the through lane and the left turn lane, the through traffic and turning traffic can be clearly distinguished from their mobile traces as they have different destination links. Thus the proposed method can be applied to the through lane and turn lane separately to estimate the queue length of each lane. On a through approach with multiple lanes, the precision of the mobile data may not be accurate enough to differentiate between different lanes, which will bring difficulty to the proposed method in this paper. This is a common challenge of all mobile data based models. The proposed method could partially solve this issue. In particular, we use trajectory reconstruction method to identify the queue location of the last sample queued vehicle in the sample mobile data, and then apply the delay based algorithm to estimate the queue length. If the exact queue lengths of two lanes are close to each other, the estimated queue length is approximately the average length of the two queues. If not, the algorithm estimates the length of the longer queues as the last queued sample is usually on the longer queue.

We next show some numerical results of testing the proposed methods in this paper. In the field test conducted in Albany, NY (Ban et al., 2011), trajectories were tracked by 9 vehicles equipped with GPS loggers. As vehicles with mobile sensors drove repeatedly over the study intersection, totally 126 traces were collected in the test, representing roughly $30 \%$ penetration of the real left turn traffic flow. The observed queue length during the test ranges from 4 to 16 . The average queue length is 7.1. As there are two major tasks in this paper: trajectory reconstruction and long queue length estimation, we will numerically test them in Section 6.1 and 6.2 respectively.

\subsection{Results of Deceleration and Acceleration Process Reconstruction}

We show the training and testing results of the trajectory reconstruction model. As there are no historical data available, the revealed traces of Type I and II vehicles are used in the training period. First for the deceleration reconstruction of a Type II vehicle, we train the model by averaging $g^{\prime}(u)$, the change of time in respect to the unit change of speed as defined in equation (8). Figure 4(a) shows the average values of $g^{\prime}(u)$ at different speeds in deceleration. In general, $g^{\prime}(u)$ increases when the speed decreases, but with significant fluctuations. Since $g^{\prime}(u)$ is the reciprocal of the deceleration rate, this figure indicates that at the beginning of the deceleration process (speed is high), the decelerations rates are large; when the vehicle slows down, the deceleration rates are reduced. This is consistent with the typical deceleration behavior in response to traffic signals (e.g., red times). Based on the calibrated $g^{\prime}(u)$ and equation (10), we are able to estimate the estimated deceleration trajectory of any vehicle given an initial point at VTL1. Figure 4(b) plots an imaginary deceleration trajectory, if the vehicle starts to decelerate at time 0 , and stops at the stop line. The estimated deceleration process of any queued vehicle in this dataset has the same shape with the curve in Figure 4(b), but with shifts in time and location.

To train the queue dissipation model in the acceleration process of a Type III vehicle, we aim to find the slope $W$ and intercept $B$ of the translated characteristic curve function (29) for any given speed. In each sub-figure in Figure 5, we plot the TIA and location coordinates of all sample vehicles at a given speed (from $1 \mathrm{ft} / \mathrm{s}$ to $20 \mathrm{ft} / \mathrm{s}$ with $1 \mathrm{ft} / \mathrm{s}$ as the increment). That is, each sub- 
figure is for a particular speed for sample vehicles during the acceleration process. Each dot in a sub-figure (e.g., that for the $1 \mathrm{ft} / \mathrm{s}$ speed) represents a vehicle trajectory point at exactly the display speed. The fitted translated characteristic line through robust linearly regression is then depicted through the data points. On top of each subplot we show the Sigma estimate (which can be considered as a robust estimate of the Root Mean Square Error) at each speed. It increases from 0.11 to 2.13 when the speed varies from $1 \mathrm{ft} / \mathrm{s}$ to $20 \mathrm{ft} / \mathrm{s}$. To address the significance of the fitting, we use $\mathrm{p} 1$ to represent the p-value of the slope $(W)$ estimate, and $\mathrm{p} 2$ to represent the pvalue of the intercept $(B)$ estimate. At relatively low speed (i.e. less than $13 \mathrm{ft} / \mathrm{s}$ ), the linear fitting passes the significance test with p-values of the coefficient estimates below 0.05 . For some higher speed, the p-value of the intercept $(B)$ estimate may exceed the threshold, but the overall performance is still acceptable for the field test dataset, considering the randomness of the driving behavior. This is particularly true for low speeds, i.e., during the initial stage of vehicle accelerations. The result validates the proposed queue dissipation model that is built on the LWR theory, as the characteristic curves are shown to be linear for the discharging process.

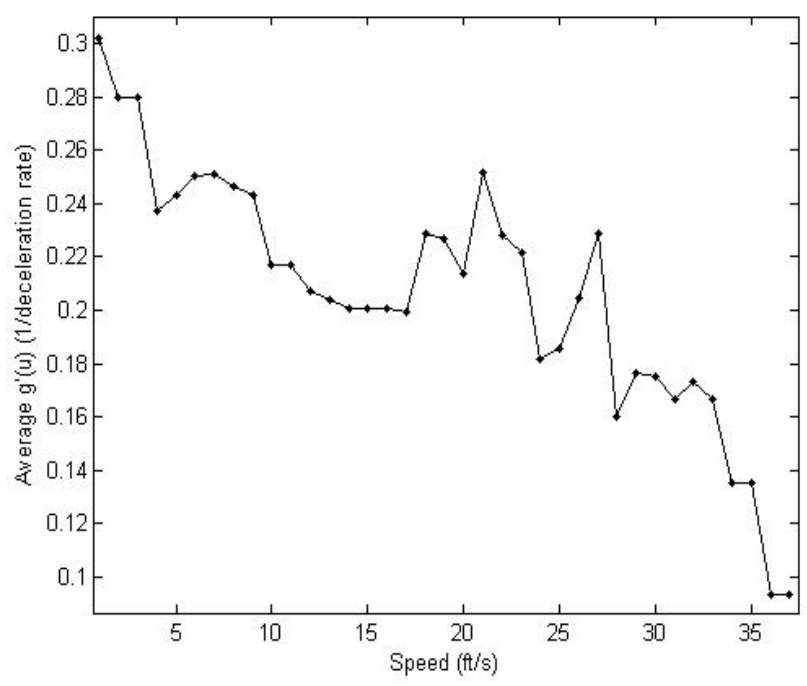

(a) Training: Average $g^{\prime}(u)$ vs. Speed

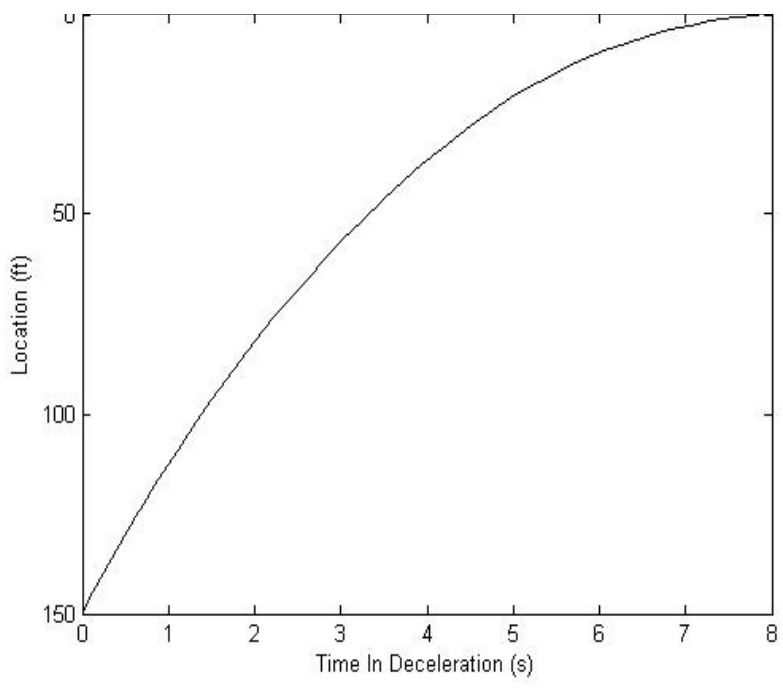

(b) Reconstructed deceleration trajectory

Figure 4 Deceleration reconstruction 

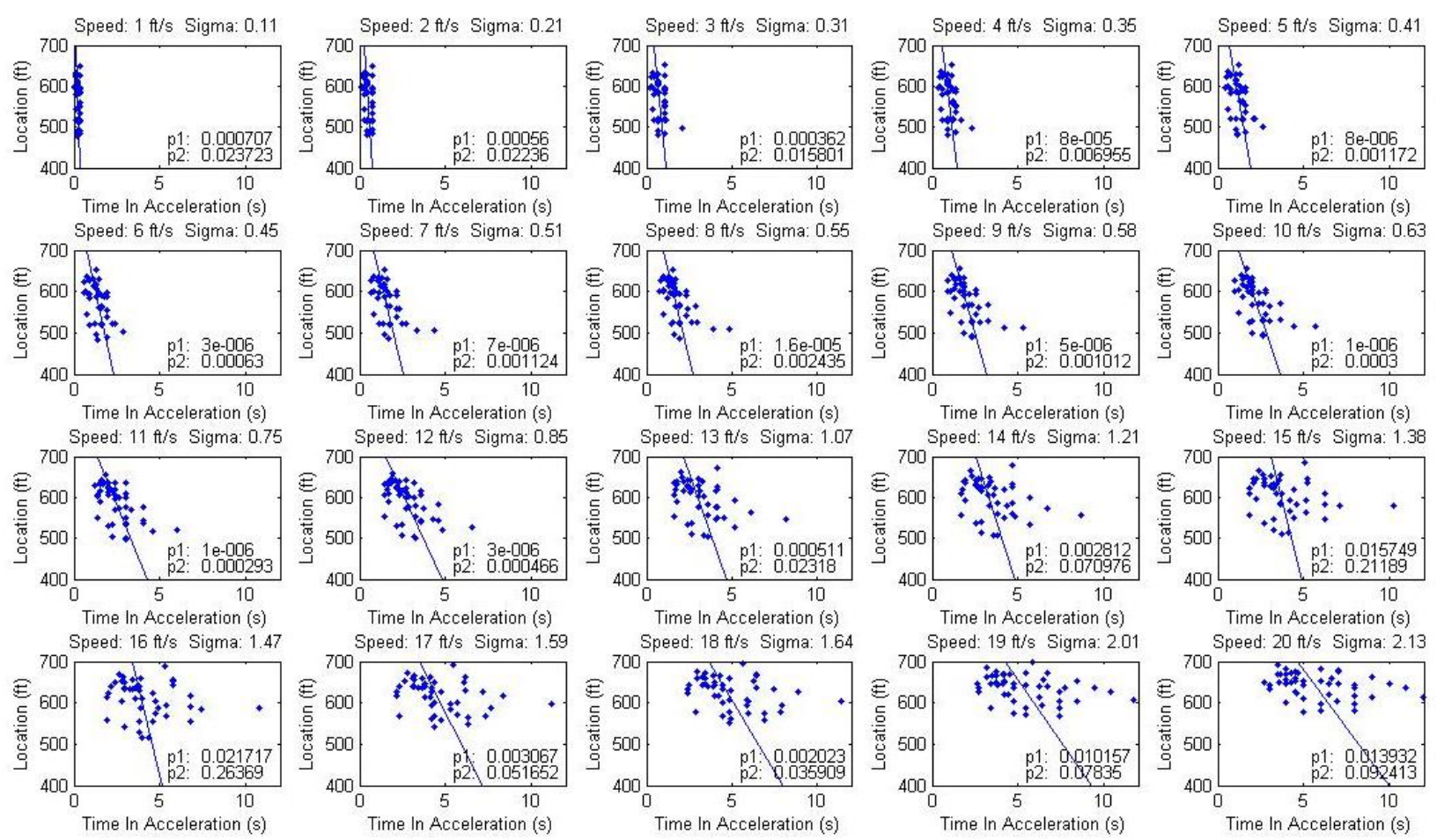

\section{Figure 5 Fitted translated characteristic line}

Based on the trained deceleration and acceleration models, we reconstruct the missing process of each vehicle. Figure 6 depicts the reconstruction results for a short time period (16:24 16:38). Figure 6(a) presents the scenario that VTL1 is closely spaced (i.e., $150 \mathrm{ft}$ ) from the stop line. The real trajectory before VTL1 is assumed to be undetected. We plot the estimated deceleration process by dotted lines, the estimated acceleration process by solid thick curves, and the real trajectory that by solid curves. We zoom in part of the trajectories around 16:26 in a window to show the details of the reconstructed traces. As shown in the figure, most of the estimated trajectories match the corresponding real ones well for both deceleration and acceleration. During the given time period, there are three moving-queue vehicles (vehicle $a, b$ and $c$ ) and two over-saturated vehicles (vehicle $d$ and $e$ ). The missing trajectories of vehicle $a$ and $b$ can be successfully reconstructed. Vehicle $c$ (Type IV) is falsely identified as a free flow vehicle since the vehicle does not have delay within the VTL zone. The observed trajectories of vehicle $d$ and $e$ cannot indicate any over-saturation, so the estimated trajectories have large deviation from the true ones. The Mean Absolute Error (MAE) in reconstructing trajectories is 3.24ft for the Type II vehicles and $8.51 \mathrm{ft}$ for the Type III vehicles. Generally the deceleration reconstruction has better performance. For the scenario in Figure 6(b), the distance between VTL1 and stop line is $350 \mathrm{ft}$. More queuing and over-saturation information are revealed by mobile sensors. Thus we have more accurate estimate on the deceleration process, including the moving-queue vehicle $c$ and over-saturated vehicles $d$ and $e$, as shown in the zoom-in window around 16:37. 
The results in Figure 6 clearly show that although the proposed trajectory reconstruction methods can correctly reconstruct missing vehicle trajectories in some cases (Type II and Type III), in other cases they cannot work well simply because the information collected in the VTL zone is not enough to detect whether a vehicle is delayed or not, and if yes whether it is oversaturated or not. In this sense, the actual location of VTL1 is still very crucial for the methods to work properly. It is recommended that field observations and engineering studies should be conducted first to have a sense of the queue size of an intersection before VTLs are deployed. For more discussions about how to properly deploy VTLs, one can refer to Hao et al. (2014a).

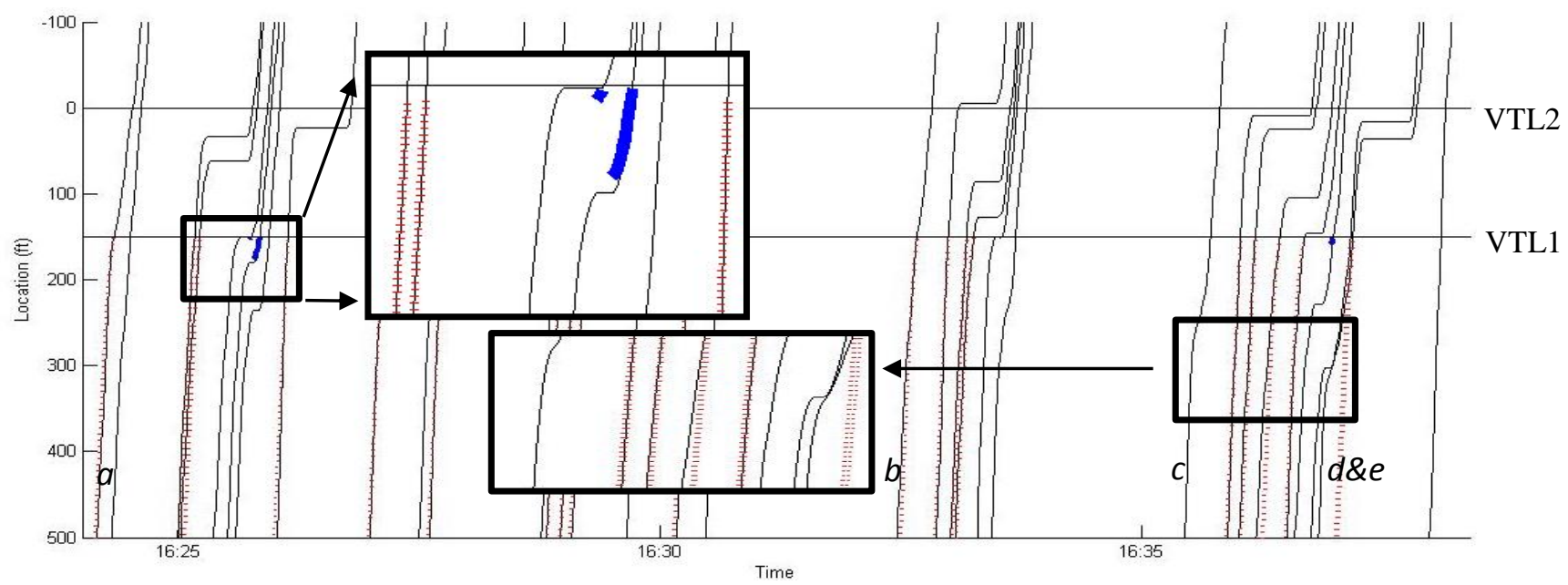

(a) $150 \mathrm{ft}$ between VTL1 and stop line

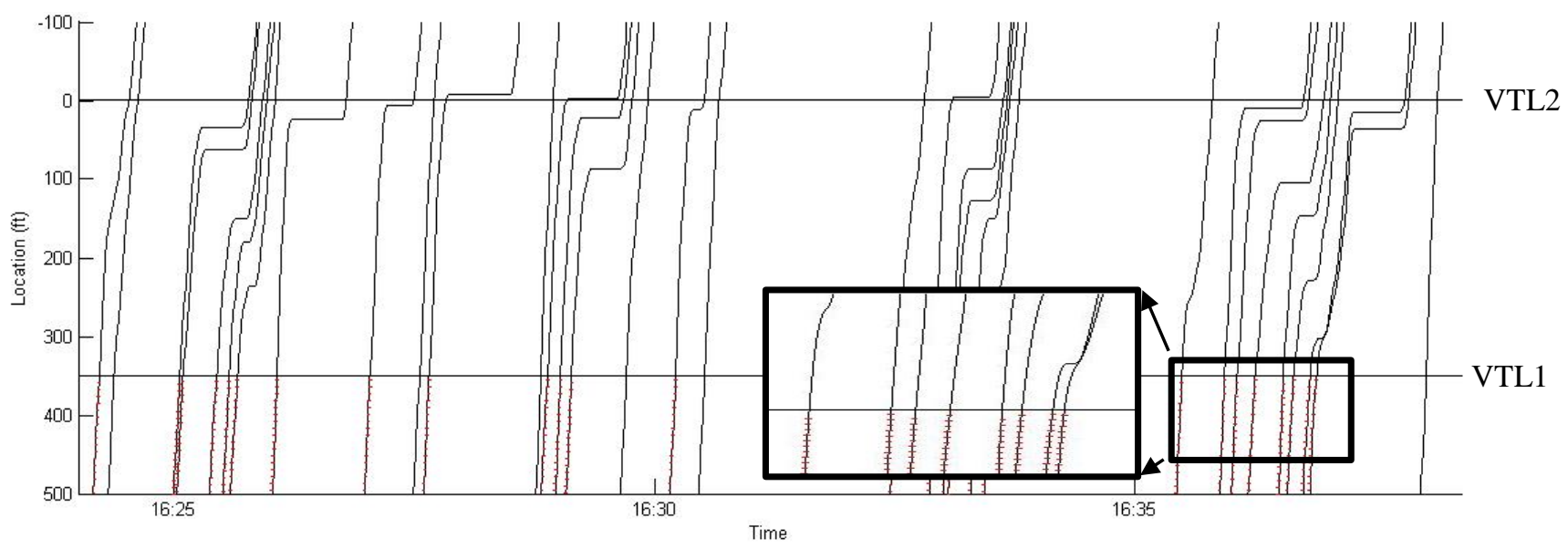

(b) VTL1 is $350 \mathrm{ft}$ from the stop line

Figure 6 Reconstructed acceleration and deceleration process

\subsection{Results of Long Queue Estimation}

In this section, we focus on the cycle-by-cycle queue profile and the maximum queue length for the scenario that VTL1 is $150 \mathrm{ft}$ from the stop line. In Figure 7, the solid dotted curves show the observed real time queue length which is well calibrated from the video data. The $x$ coordinates 
of the dots represent the observed times that the queued vehicles join the queues, and the $y$ coordinates of the dots represent the queue positions of those queued vehicles. The thick dashed curves with crosses represent the queue position profiles and maximum queue lengths estimated by the proposed method. The bottom data point in each dashed curve indicates the estimated time that the first queue vehicle in the cycle joins the queue, and the top data point indicates the maximum queue length. The other data points show the queue position and queue time of each sample queued vehicle in the cycle. We also depict the result of the short queue length estimation in Ban et al. (2011) using dot-dashed lines. As shown in the figure, 50\% of the cycles have at least one queued sample so that the long queue estimation method proposed in this paper can be successfully applied, which is defined as the success rate of the method in Ban et al. (2011). As the proposed method requires fewer samples in a cycle to estimate the queue length, it outperforms Ban's method that only has $28 \%$ success rate for the same dataset. Within each cycle, the estimated curve using the proposed method in the paper can capture the growing trend and the actual shape profile of the queue length well.

The observed and estimated maximum queue length of all the cycles with at least one queued sample are also summarized in Table 1. From this table, one can compare the estimation performance of queue lengths by both methods in each cycle. To measure the performance of maximum queue length estimation, we compute the Mean Absolute Error (MAE) which is defined as:

$$
M A E=\frac{\sum_{i=1}^{N}\left|Q_{i}^{*}-\bar{Q}_{i}\right|}{N}
$$

Here $Q_{i}^{*}$ is the estimated maximum queue length of the $\mathrm{i}^{\text {th }}$ cycle, $\bar{Q}_{i}$ is the true value, and $N$ is the total number of cycles that have at least one queued sample. The MAE of the proposed method is 1.61, which is much better than 3.79, the MAE of the short queue model in Ban et al. (2011).

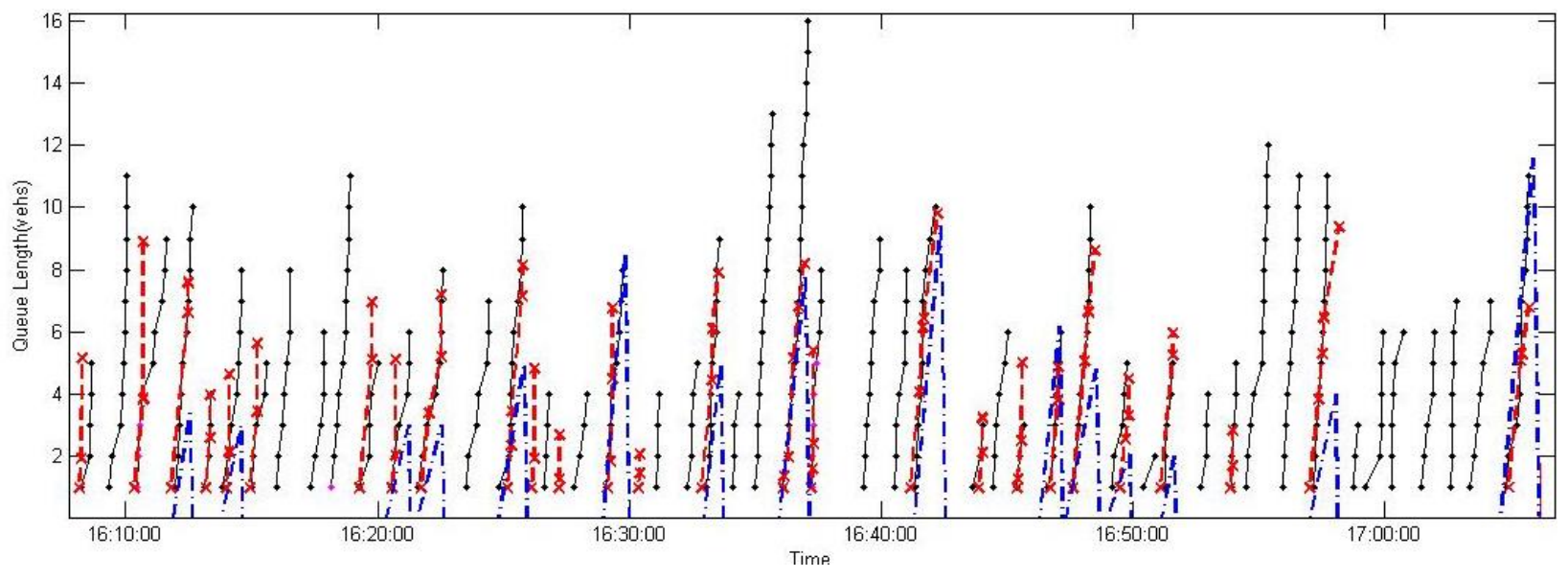

Figure 7 Cycle-by-cycle long queue estimation 
Table 1 Observed and estimated maximum queue lengths

\begin{tabular}{|c|c|c|c|c|c|}
\hline Cycle ID & $\begin{array}{c}\text { Observed } \\
\text { queue length } \\
\text { (veh) }\end{array}$ & $\begin{array}{l}\text { Estimated queue } \\
\text { length by proposed } \\
\text { method (veh) }\end{array}$ & $\begin{array}{l}\text { Absolute error } \\
\text { by proposed } \\
\text { method (veh) }\end{array}$ & $\begin{array}{l}\text { Estimated queue } \\
\text { length by short } \\
\text { queue model (veh) }\end{array}$ & $\begin{array}{c}\text { Absolute error by } \\
\text { short queue model } \\
\text { (veh) }\end{array}$ \\
\hline 1 & 5 & 5.14 & 0.14 & & \\
\hline 2 & 9 & 8.92 & 0.08 & & \\
\hline 3 & 10 & 7.60 & 2.40 & 3.39 & 6.61 \\
\hline 4 & 4 & 3.99 & 0.01 & & \\
\hline 5 & 8 & 4.63 & 3.37 & 3.00 & 5.00 \\
\hline 6 & 5 & 5.65 & 0.65 & & \\
\hline 7 & 5 & 6.96 & 1.96 & & \\
\hline 8 & 6 & 5.12 & 0.88 & 3.00 & 3.00 \\
\hline 9 & 8 & 7.12 & 0.88 & 3.00 & 5.00 \\
\hline 10 & 10 & 8.16 & 1.84 & 5.00 & 5.00 \\
\hline 11 & 4 & 4.84 & 0.84 & & \\
\hline 12 & 4 & 2.71 & 1.29 & & \\
\hline 13 & 8 & 6.78 & 1.22 & 8.57 & 0.57 \\
\hline 14 & 4 & 2.09 & 1.91 & & \\
\hline 15 & 9 & 7.89 & 1.11 & 5.00 & 4.00 \\
\hline 16 & 16 & 8.20 & 7.80 & 7.77 & 8.23 \\
\hline 17 & 8 & 5.41 & 2.59 & & \\
\hline 18 & 10 & 9.79 & 0.21 & 9.39 & 0.61 \\
\hline 19 & 3 & 3.24 & 0.24 & & \\
\hline 20 & 3 & 5.01 & 2.01 & & \\
\hline 21 & 6 & 4.89 & 1.11 & 6.17 & 0.17 \\
\hline 22 & 10 & 8.60 & 1.40 & 4.79 & 5.21 \\
\hline 23 & 5 & 4.51 & 0.49 & 2.08 & 2.92 \\
\hline 24 & 5 & 5.98 & 0.98 & 2.00 & 3.00 \\
\hline 25 & 5 & 2.85 & 2.15 & & \\
\hline 26 & 11 & 9.37 & 1.63 & 4.00 & 7.00 \\
\hline 27 & 11 & 6.79 & 4.21 & 11.59 & 0.59 \\
\hline MAE (veh) & & & 1.61 & & 3.79 \\
\hline
\end{tabular}

We further test the distance effect on the long queue estimation performance. In this test we measure the MAE and success rate for different locations of VTL1. The distance between VTL1 and the stop line varies from $50 \mathrm{ft}$ to $500 \mathrm{ft}$. As shown in Figure 8, generally the performance becomes better when we move VTL1 farther away from the stop line. That is because a longer distance between VTL1 and the stop liner reveals more vehicle trajectories and queue information. If VTL1 is quite close to the stop line (i.e. $50 \mathrm{ft}$ ), the MAE is about 2.2 and the success rate is only $35 \%$. When we move the VTL1 to $200 \mathrm{ft}$, the MAE drops to 1.54 and the success rate reaches $56 \%$. For the longer distance, the positive effect of distance is not that significant because most vehicles are queued within the range of $200 \mathrm{ft}$ from the stop line. As shown in Figure 8(a) and (b), MAE is floating around 1.55 for larger distances, while the success rate stays at the maximum value 58\%. The results in Figure 8 further indicate that the actual location of VTL1 is critical. One the one hand, it needs to be deployed relatively far from the stop line to capture vehicle queuing processes. On the other hand, deploying VTL1 too far away from the stop line would not benefit much for queue length estimation purposes. A tradeoff is 
clearly needed here which will has to be based on the actual observations of the traffic conditions of the intersection.

This distance effect on estimation performance can be further explained by Table 2, which lists the percentage of each vehicle type at each distance, including moving-queue vehicles (labeled as "M") and over-saturated vehicles (labeled as "O"). When VTL1 is moved farther away from the stop line (i.e., further upstream), the percentage of Type I vehicle increases and the percentage of Type IV vehicle decreases. There are less Type II vehicles when VTL1 is too close to or too far from the stop line. The Type III vehicles have similar trend as the Type II, but the percentage is much lower. Furthermore, when VTL1 is too close to the stop line (less than 200 $\mathrm{ft}$ ), no moving-queue or over-saturated vehicle can be detected. As VTL1 gets farther away from the stop line, moving-queue vehicles can be detected first (larger than $250 \mathrm{ft}$ ) and then oversaturated vehicles (larger than $350 \mathrm{ft}$ ). In addition, as shown in this table, the total percentage of Type I, II and III vehicles significantly impact the performance on long queue estimation. Their total percentage increases from $25 \%$ to $60 \%$ when the VTL1 distance increase from 50ft to 200ft, and keeps at the same level when VTL1 is moved farther away. Correspondingly in Figure 8, we can observe a dramatic performance enhancement from $50 \mathrm{ft}$ to $200 \mathrm{ft}$, and stable performance afterwards.

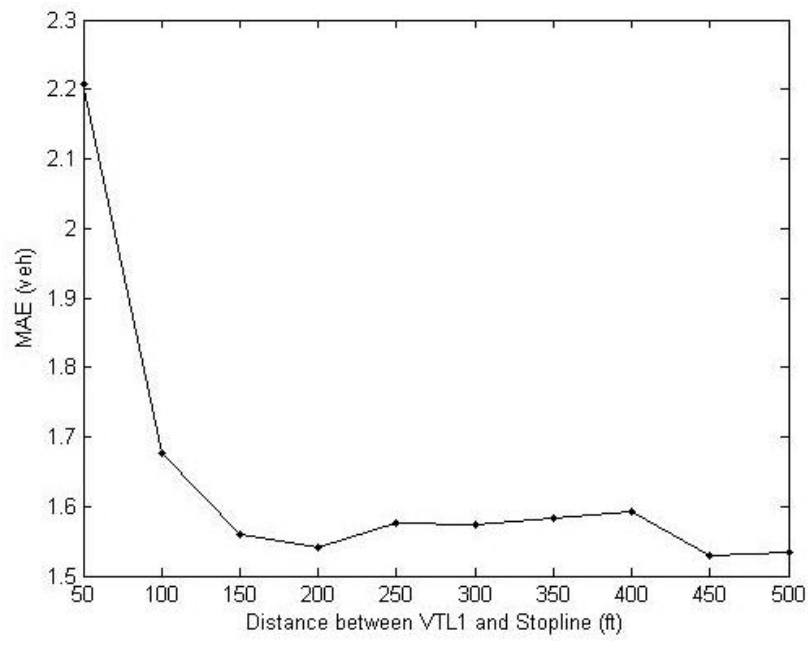

(a) MAE vs. Distance

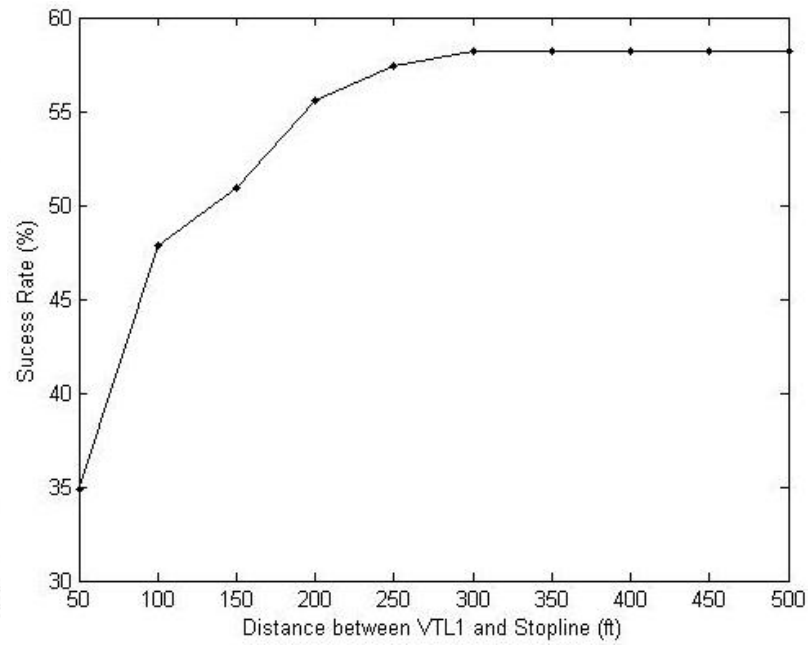

(b) Success rate vs. Distance

Figure 8 Distance effect on performance

Table 2 Distance effect on percentage of vehicle type

\begin{tabular}{|c|c|c|c|c|c|c|c|c|c|c|c|}
\hline \multicolumn{2}{|c|}{ Distance $(\mathrm{ft})$} & 50 & 100 & 150 & 200 & 250 & 300 & 350 & 400 & 450 & 500 \\
\hline \multirow{4}{*}{$\begin{array}{c}\text { Percentage } \\
\text { of vehicle } \\
\text { type } \\
(\%)\end{array}$} & I & 0 & 0 & 1 & 5 & 14 & 23 & 28 & 34 & 36 & 39 \\
\hline & II & 22 & 32 & 44 & 50 & 45 & 38 & 32 & 26 & 24 & 21 \\
\hline & III & 3 & 7 & 9 & 5 & 4 & 1 & 0 & 0 & 0 & 0 \\
\hline & IV & 76 & 61 & 46 & 30 & 21 & 21 & 21 & 21 & 19 & 19 \\
\hline
\end{tabular}




\begin{tabular}{|r|r|r|r|r|r|r|r|r|r|r|r|}
\hline & $\mathrm{M}$ & 0 & 0 & 0 & 10 & 16 & 18 & 18 & 17 & 19 & 19 \\
\cline { 2 - 11 } & $\mathrm{O}$ & 0 & 0 & 0 & 0 & 0 & 0 & 2 & 2 & 2 & 2 \\
\hline
\end{tabular}

\section{Conclusions}

In this paper, we proposed long queue estimation models using short vehicle traces obtained from mobile sensors, which consist of two major steps: trajectory reconstruction and delay-based queue length estimation. To estimate the missing acceleration/deceleration process before VTL1, we developed models based on classic traffic flow theory, i.e. the simplified car following theory for deceleration reconstruction and the LWR theory for acceleration reconstruction. We selected vehicle speeds as the key measure in those models, as it can be directly measured using mobile sensors, which also have close relations with other variables such as locations and travel times. To reduce the impact of low penetration rates that are still a primary problem in practice, we aggregated the sparse data over multiple cycles and calibrated the deceleration/acceleration models. The reconstructed trajectories can transform the long queue problem into a short queue problem, by recovering the free flow arrival process after the trajectory reconstruction. The delay-based queue length estimation model was then developed to estimate the queue length profile and the maximum queue length of each cycle. This method is simple but effective, especially when dealing with low penetration conditions since only one queued sample is required to estimate the queue length. As the method works without knowing signal timing information, it is applicable to pretimed, actuated, and adaptive signals. The proposed long queue model also significantly improved both the success rate and the estimation error for the field data in the Albany, NY test, compared with the previous method in Ban et al. (2011).

The limitations of the proposed methods and several future research directions are summarized as follows:

(1) In this paper, we trained the model using a one hour field dataset, which is still too small. More field tests should be conducted to validate and calibrate the proposed long queue model.

(2) The methods proposed in the paper can work for single-lane intersections. For multi-lane intersections, one needs to properly distinguish lanes using mobile data. However, data errors especially in urban areas (such as urban canyon if GPS devices are considered) may pose great challenges. Future research is needed to conduct a more comprehensive investigation about how the proposed methods can be extended to multi-lane intersections in urban areas. In addition, long queues in the real world usually company with lane merging and splitting. The authors will investigate those complicated cases using mobile data and video camera data (if available), and incorporate them in the queue length estimation methods.

(3) Under heavily congested conditions, the queue may spillover to the upstream intersection. This would be considered as an extreme long queue case. Detecting and measuring the 
impact of this spillover issue is also an important future research topic. For this, the research work by Wu et al. (2011) based on loop detector data may provide useful insight.

(4) The proposed method requires high precision mobile data as input when identifying deceleration/acceleration processes and measuring queue locations. In real world the mobile data may not have the ideal precision. In this case, robust algorithms are needed to process data with noise or errors.

(5) In this paper, we develop the long queue model based on GPS data of high sampling frequency, e.g., in the field test, data were recorded every second. The proposed method may fail to work if the sampling rate is too large, e.g., 15 or 30 seconds. However, similar reconstruction methods may still be developed to deal with mobile data of low sampling frequency, which will be pursued in future research.

(6) We focused on queue length estimation in this paper, by post-processing the collected mobile data, for the purpose of measuring arterial performances. Accurate prediction of how queue length will vary in short term (say the next five minutes or so) will be more useful for traffic operations such as signal control and optimization. This may be pursued in future research.

\section{Acknowledgement}

The authors would like to thank the three anonymous referees for their insightful comments and helpful suggestions on earlier versions of the paper. This research is partially funded by the National Science Foundation (NSF) grant CMMI-1055555. Any opinions, findings, and conclusions or recommendations expressed in this paper are those of the authors and do not necessarily reflect the views of the NSF.

\section{References}

1. Balke, K., Charara, H., \& Parker, R. (2005). Development of a Traffic Signal Performance Measurement System (TSPMS) (Report 0-4422-2). College Station, TX: Texas Transportation Institute.

2. Ban, X., Herring, R., Hao, P., \& Bayen, A. (2009). Delay pattern estimation for signalized intersections using sample travel times. Transportation Research Record, 2130, 109-119.

3. Ban, X., Hao, P., \& Sun, Z. (2011). Real time queue length estimation for signalized intersections using sample travel times from mobile sensors. Transportation Research Part C, 19(6), 1133-1156.

4. Brindle, B. (2014). How does Google Maps predict traffic? http://electronics.howstuffworks.com/how-does-google-maps-predict-traffic.htm. Access on June 17, 2015.

5. Cheng, Y., Qin, X., Jin, J., \& Ran, B. (2012). An exploratory shockwave approach to estimating queue length using probe trajectories. Journal of Intelligent Transportation Systems, 16(1), 12-23.

6. Comert, G. (2013). Simple analytical models for estimating the queue lengths from probe vehicles at traffic signals. Transportation Research Part B 55(1), 59-74 
7. Comert, G., \& Cetin, M. (2009). Queue length estimation from probe vehicle location and the impacts of sample size. European Journal of Operational Research 197(1), 196202.

8. Daganzo, C., (2005). A variational formulation of kinematic waves: basic theory and complex boundary conditions. Transportation Research Part B 39 (2), 187-196.

9. Daganzo, C., (2005). A variational formulation of kinematic waves: Solution methods. Transportation Research Part B 39 (10), 934-950.

10. Daganzo, C., (2006). On the variational theory of traffic flow: well-posedness duality and applications. Networks and Heterogeneous Media 1 (4), 601-619.

11. Hao, P., Ban, X., Bennett, K., Ji, Q., \& Sun, Z. (2012). Signal timing estimation using sample intersection travel times. IEEE Transactions on Intelligent Transportation Systems, 13(2), 792-804.

12. Hao, P., Sun, Z., Ban, X., Guo, D., \& Ji, Q. (2013). Vehicle index estimation for signalized intersections using sample travel times. Transportation Research, Part C 36, 513-529.

13. Hao, P., Ban, X., \& Yu, J. (2014a). Estimation of queue location for signalized intersections using sample travel times from mobile sensors. Journal of Intelligent Transportation Systems, in press.

14. Hao, P., Ban, X., Guo, D., \& Ji, Q. (2014b). Cycle-by-cycle queue length distribution estimation using sample travel times. Transportation Research Part B 68, 185-204

15. Hoh, B., Gruteser, M., Herring, R., Ban, J., Work, D., Herrera, J.C., \& Bayen, A. (2008). Virtual trip lines for distributed privacy-preserving traffic monitoring. Proceedings of the Sixth Annual International conference on Mobile Systems, Applications and Services (MobiSys 2008), 15-28.

16. Hunt, P. B., Robertson, D. I., Bretherton, R. D. \& Winton, R. I. (1981). SCOOT - a traffic responsive method of co-ordinating signals. TRL Laboratory Report 1014.

17. Izadpanah, P., Hellinga, B., \& Fu, L. (2009). Automatic traffic shockwave identification using vehicles' trajectories [CD]. Proceedings of the 88th Annual Meeting of the Transportation Research Board, Washington, DC.

18. Lighthill, M.J., \& Whitham, G.B. (1955). On kinematic waves I: Flood movement in long rivers. II: A theory of traffic flow on long crowded roads. Proceedings of Royal Society (London) A229, 281-345.

19. Liu, X., \& Ma, W. (2008). A real-time performance measurement system for arterial traffic signals [CD]. Proceedings of the 87th Annual Meeting of Transportation Research Board, Washington, DC.

20. Liu, X., Wu, X., Ma, W., \& Hu, H. (2009). Real time queue length estimation for congested signalized intersections. Transportation Research, Part C, 17(4). 412-427.

21. Ma, W. (2008). A real-time performance measurement system for arterial traffic signals (Doctorial dissertation, University of Minnesota, Twin Cities). Retrieved from http://www.ce.umn.edu/ liu/Thesis/Ma_Dissertation_Final.pdf

22. Newell, G.F. (1965). Approximation methods for queues with application to the fixedcycle traffic light. SIAM Review, 7, 223-240.

23. Newell, G.F., (1993). A simplified theory of kinematic waves in highway traffic, part I: General theory. Transportation Research, Part B, 27(4), 281-287. 
24. Newell G.F. (2002). A simplified car-following theory: a lower order model. Transportation Research, Part B, 36(3), 195-205.

25. Richards, P.I. (1956). Shock waves on the highway. Operations Research, 4(1), 42-51.

26. Skabardonis, A., \& Geroliminis, N. (2008). Real-time monitoring and control on signalized arterials. Journal of Intelligent Transportation Systems, 12 (2), 64-74.

27. Smaglik, E. J., Sharma, A., Bullock, D.M., Sturdevant, J.R. \& Duncan, G. (2007). EventBased data collection for generating actuated controller performance measures. Transportation Research Record, 2035, 97-106.

28. Stephanopoulos, G., Michalopoulos, P.G., \& Stephanopoulos, G. (1979). Modelling and analysis of traffic queue dynamics at signalized intersections. Transportation Research, Part A. 13(5), 295-307.

29. Sun, Z. Zan, B., Ban, X., \& Gruteser, M. (2013). Privacy protection method for finegrained urban traffic modeling using mobile sensors. Transportation Research Part B 56(1), 50-69.

30. Webster, F.V. (1958). Traffic signal settings (Road Research Laboratory Technical Paper No. 39). London, UK: HMSO.

31. Wu, X., Liu, X., \& Geroliminis, N. (2011). An empirical analysis on the arterial fundamental diagram. Transportation Research, Part B, 45(1), 255-266.

32. Zan, B., Hao, P., Gruteser, M., \& Ban, X. (2011). VTL zone-based path cloaking algorithm. Proceedings of the 14th International IEEE Conference on Intelligent Transportation Systems, 1525 - 1530. 


\section{Appendix: Long queue model for moving-queue and over-saturated vehicles}

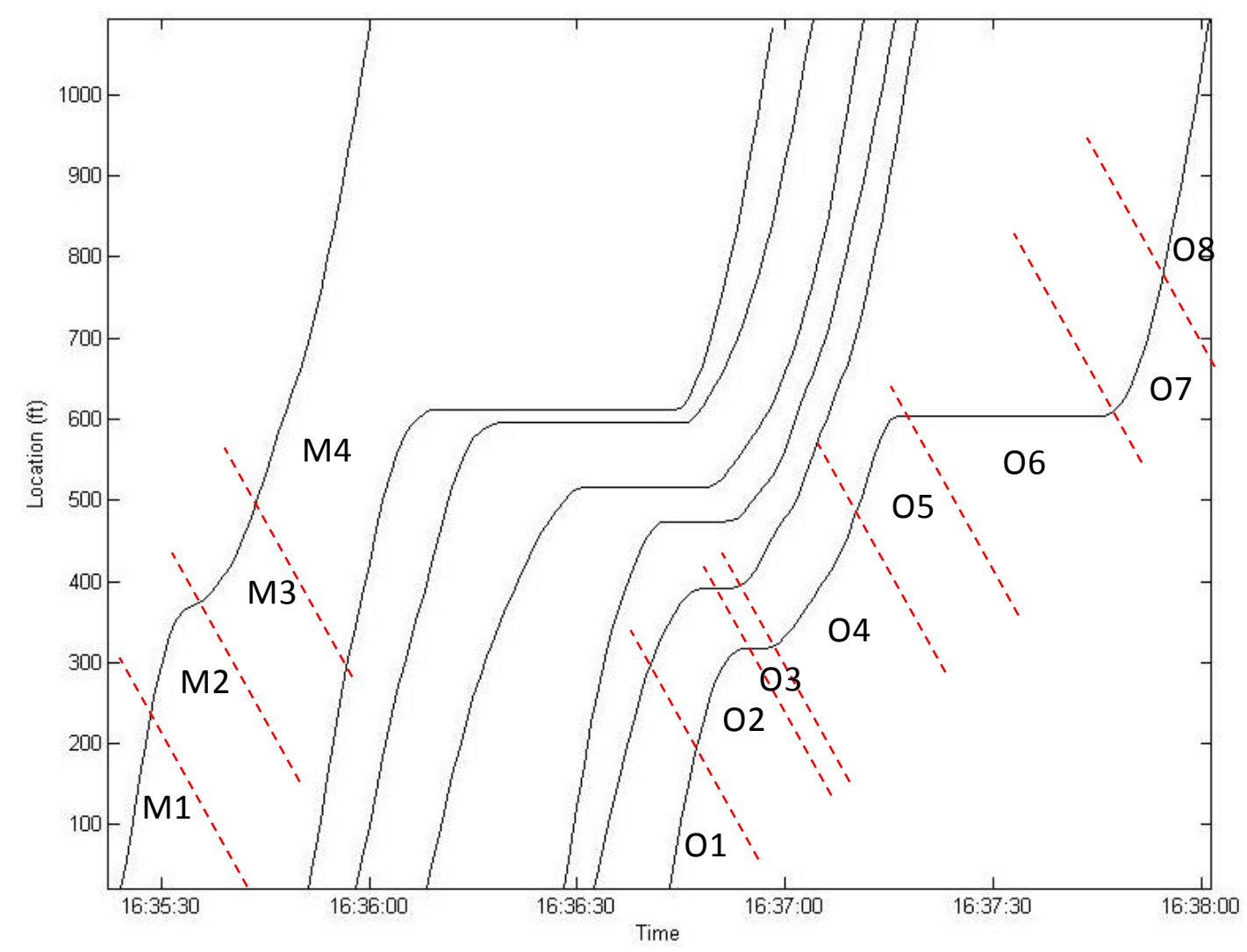

Figure 9 Moving queues and over-saturated vehicles in long queue model

\section{Moving Queues}

Vehicles in moving queues are defined as the vehicles that are "affected but not fully stopped by the standing queue" (Ban et al., 2011). As shown in Figure 9, the trajectory of a moving-queue vehicle consists of four processes: arriving (M1), deceleration (M2), acceleration (M3) and departure (M4). For the deceleration process M2, we assume that the moving-queue vehicle follows the same trajectory of leading vehicles with shifts in time and space, until the queue in front is cleared. Then this vehicle accelerates to free flow speed. The transition point between $\mathrm{M} 2$ and M3 is the moving queue point (MQP) of a moving-queue vehicle, at which the speed of the vehicle drops to the minimum (but greater than the queuing speed, i.e. $1 \mathrm{ft} / \mathrm{s}$ ).

The moving queue can be considered as the intermediate state between the standing queue and the free flow traffic. In the long queue model we use estimated intersection delay to distinguish the moving-queue vehicles from the free flow vehicles. If the vehicle passes VTL1 before decelerating, the measured delay is exactly the same as the intersection delay. If the vehicle passes VTL1 when decelerating, the total intersection delay can be estimated by the deceleration process reconstruction method in Section 4. If the vehicle passes VTL1 when accelerating, we 
approximately estimate the intersection delay by assuming that the vehicle finishes its deceleration process right before entering the VTL zone. A reconstructed deceleration process is added to the trajectory within the VTL zone based on the speed at VTL1. We then do a queuing state test based on the measured/estimated intersection delay. If the vehicle's estimated intersection delay is greater than a predefined threshold, i.e. minimum traverse time, the vehicle is identified as a queued vehicle. Otherwise, the vehicle is a free flow vehicle.

For a queued vehicle that passes the queuing state test, following criteria will be applied to determine whether this vehicle is a standing queue vehicle or a moving-queue vehicle. We check the minimum speed for the vehicle trajectory from VTL1 to the stop line. If that speed is lower than the queuing speed threshold, i.e. $1 \mathrm{ft} / \mathrm{s}$, this vehicle is identified as a standing queue vehicle. If this vehicle has its minimum speed at the beginning of the trajectory within the VTL zone, and keep accelerating afterwards, it could be either a standing queue vehicle or a moving-queue vehicle, depending on whether it stops before VTL1. For this case, we take this vehicle as a Type III vehicle and apply the shockwave-based queue dissipation model in Section 5 to reconstruct the acceleration process. The queue location is then estimate to help identify the maximum queue length, as stated in Section 5.5. If we find the minimum speed point somewhere in the middle of the trajectory, with the speed that is higher than $1 \mathrm{ft} / \mathrm{s}$, the vehicle is identified as a moving queue vehicle and that point is the MQP. The queue length is then estimated based on equation (2), which requires intersection delay and queue location as the input. The intersection delay is already measured or estimated in the queuing state test, but the queue location is not that easy to evaluate, as moving-queue vehicles do not actually stack with jam density. In this paper we estimate the queue location by imaging that the moving queue will fully stop in an "equivalent standing queue" which is defined in Ma (2008). We use acceleration rebuilding method in Section 5 based on the speed at MQP to trace this equivalent queue location of the moving-queue vehicle. Finally, we use the equivalent queue location to estimate the maximum queue length using equation (35).

\section{Over-saturation}

Under heavily congested conditions, some vehicles may not be cleared within one cycle. Here we discuss the long queue estimation method for those over-saturated vehicles that experience extra delays. As shown in Figure 9, the example over-saturated vehicle experiences two stopand-go periods. This vehicle arrives with free flow state (O1). In the first queuing period, it decelerates $(\mathrm{O} 2)$, queues $(\mathrm{O} 3)$ and accelerates $(\mathrm{O} 4)$ until the signal turns red again and the residual queue spills back. Then the vehicle experience the second queuing period which starts with another deceleration (O5), succeeded with queuing (O6), acceleration (O7) and departure (O8) process. If the vehicle passes VTL1 during the second period, i.e. O5-O8, one cannot distinguish this over-saturated vehicle with a normally queued vehicle. In this case, we consider this vehicle as a normally queued vehicle, and estimate the queue length using the model presented in the previous section. If the vehicle passes VTL1 during the first period, the short 
trajectory can identify the existence of over-saturation, and indicate the queue information of the cycle it arrives and the cycle it leaves. In this section, we use Type O-I, O-II, O-III to represent the scenarios when the vehicle passes VTL1 in the processes of the first period (i.e. O1, O2, O4) respectively. Note that the vehicle cannot pass the VTL when it is in the queuing process O3.

First we focus on Type O-I. This is actually a short queue over-saturation case because all queuing information of the vehicle is measured. We can estimate the queue lengths of both cycles using the method of Ban et al. (2011) for over-saturation case, but with constant arrival headway assumption. According to the definition in Ban et al. (2011), the intersection delay of an over-saturated vehicle consists of two components: the queuing delay in the first cycle and the over-saturation delay in the second cycle. Correspondingly, we define the delay in the first period as the queuing delay, and the delay in the second period as the over-saturation delay in this paper. As the arrival rates of both cycles are assumed to be constant, behind the study vehicle, the total intersection delays of the vehicles which depart at the second cycle drops linearly from $t_{S L}-t_{N A T}$ to zero. Thus the queue length estimation equation for the normal case is applied to figure out the second cycle.

$$
Q_{2}=k_{j} L_{2}+\frac{t_{S L}-t_{N A T}}{h_{a}-h_{s}}+1
$$

To estimate the queue length of the first cycle, we additionally measure the time (defined as $t_{O 4}$ ) and location (defined as $x_{O 4}$ ) of the end of acceleration process in the first queuing period (i.e. O4). The queuing delay with process $\mathrm{O} 2-\mathrm{O} 4$ is then computed as

$$
\tau_{O 4}=\left(t_{O 4}-t_{f}\right)-\frac{x_{f}-x_{O 4}}{u_{f}}
$$

As the delay in the first cycle decreases with constant reduction rate $\frac{1}{h_{a}-h_{s}}$ in term of the vehicle position, the queue length of the first cycle is

$$
Q_{1}=k_{j} L_{1}+\frac{\tau_{O 4}}{h_{a}-h_{s}}+1
$$

The Type O-II is similar to the previous case, but with incomplete deceleration process. In this case the no-delay arrival time $t_{N A T}$ can be estimated using the deceleration reconstruction method in Section 4. The queue lengths of both cycles can be then estimated via equations (37)(39). For Type O-III, the queue position of the over-saturated vehicle in the first cycle is estimated using the acceleration rebuilding algorithm as mentioned in Section 5. This estimated queue position provides a valid lower bound when estimating the queue length of the first cycle. 